\title{
Use of Dirichlet Distributions and Orthogonal Projection Techniques for the Fluctuation Analysis of Steady-State Multivariate Birth-Death Systems
}

\author{
Filippo Palombi ${ }^{\mathrm{a} *}$ and Simona Toti ${ }^{\mathrm{b}}$ \\ ${ }^{a}$ ENEA - Italian Agency for New Technologies, Energy and \\ Sustainable Economic Development, Via Enrico Fermi 45, 00040 Frascati - Italy \\ ${ }^{\mathrm{b}}$ ISTAT - Istituto Nazionale di Statistica, Via Cesare Balbo 16, 00184 Rome - Italy
}

July 2014

\begin{abstract}
Approximate weak solutions of the Fokker-Planck equation represent a useful tool to analyze the equilibrium fluctuations of birth-death systems, as they provide a quantitative knowledge lying in between numerical simulations and exact analytic arguments. In the present paper, we adapt the general mathematical formalism known as the Ritz-Galerkin method for partial differential equations to the Fokker-Planck equation with time-independent polynomial drift and diffusion coefficients on the simplex. Then, we show how the method works in two examples, namely the binary and multistate voter models with zealots.
\end{abstract}

\section{Introduction}

Multivariate birth-death models have since long captured the interest of researchers in statistical physics as they represent a natural mathematical framework to investigate a plethora of interdisciplinary problems, ranging from opinion diffusion to language emergence, cultural dissemination and epidemic spreading [1, 2]. Broadly speaking, such models describe an evolving population of agents, each lying in one of $\mathrm{Q}$ allowed physical states. The system is macroscopically represented by a state vector $\phi=\left(\phi_{k}\right)_{k=1}^{\mathrm{Q}}$, with $\phi_{\mathrm{k}}$ denoting the fraction of agents in the $\mathrm{k}$-th state. By definition, for $\mathrm{Q}<\infty$ the state vector lives on the $\mathrm{Q}-$ simplex

$$
\mathrm{T}_{\mathrm{Q}}(\mathrm{s})=\left\{\phi \in \mathbb{R}_{+}^{\mathrm{Q}}: \quad \sum_{\mathrm{k}=1}^{\mathrm{Q}} \phi_{\mathrm{k}}=\mathrm{s}\right\} .
$$

The meaning of the parameter $s$ will become clear in the sequel, while for the time being the reader may assume $s=1$. If the microscopic dynamics of the model is determined by Markovian agent-agent

*Corresponding author. E-mail: filippo.palombi@enea.it 
interactions altering the components of $\phi$, then in the thermodynamic limit the system is known to obey a Fokker-Planck equation (FPE) (see for instance ref. [3, chapt. 7]),

$$
\begin{aligned}
& \partial_{t} \mathcal{P}(t, \bar{\phi})=-\sum_{k=1}^{Q-1} \partial_{k}\left[A_{k}(t, \bar{\phi}) \mathcal{P}(t, \bar{\phi})\right]+\frac{1}{2} \sum_{i, k=1}^{Q-1} \partial_{i} \partial_{k}\left[B_{i k}(t, \bar{\phi}) \mathcal{P}(t, \bar{\phi})\right] \equiv \mathcal{L}_{F P} \cdot \mathcal{P}(t, \bar{\phi}), \\
& \mathcal{P}(t, \bar{\phi})=0 \quad \text { if } \quad \phi \notin T_{Q}(s) .
\end{aligned}
$$

where $\bar{\phi}=\left(\phi_{k}\right)_{k=1}^{\mathrm{Q}-1}$ denotes the essential state vector (obtained from $\phi$ by conventionally leaving out its Q-th component), $\mathcal{P}(t, \bar{\phi})$ represents the probability density of $\bar{\phi}$ at time $t$ and we define $\partial_{t} \equiv \partial / \partial t$ and $\partial_{k} \equiv \partial / \partial \phi_{k} . \mathcal{L}_{F P}$ is commonly referred to as the Fokker-Planck operator. We do not impose any initial condition to eq. 1.2, as this is not relevant to our aims.

We shall make the assumption - valid for several birth-death models - that the drift coefficients $\left(A_{k}\right)_{k=1}^{Q-1}$ and the diffusion ones $\left(B_{i k}\right)_{i, k=1}^{Q-1}$ are time-independent polynomials of the components of $\bar{\phi}$. We shall also assume that the stochastic dynamics of the model has no exit states such as consensus or no-infected-agents configurations. If this the case, the system is expected to asymptotically relax to a dynamic equilibrium, with $\phi$ wandering across $T_{Q}(s)$ according to the stochastic process

$$
\mathrm{d} \phi_{\ell}(\mathrm{t})=\mathrm{A}_{\ell}(\bar{\phi}) \mathrm{d} \mathrm{t}+\sum_{\mathrm{k}=1}^{\mathrm{Q}-1} \mathrm{C}_{\ell k}(\bar{\phi}) \mathrm{d} W_{\mathrm{k}}(\mathrm{t})+\mathrm{dK}_{\ell}(\mathrm{t}), \quad \ell=1, \ldots, \mathrm{Q}-1,
$$

and eventually distributing according to a limit probability density $\mathcal{P}(\bar{\phi})=\lim _{t \rightarrow \infty} \mathcal{P}(t, \bar{\phi})$. Here, the matrix $C$ is related to the diffusion matrix via $B=C \cdot C^{T}\left(C^{T}\right.$ is the transposed matrix of $\left.C\right)$, $W(t)=\left(W_{k}(t)\right)_{k=1}^{Q-1}$ is a Wiener process describing the stochastic diffusion of the state vector and $\mathrm{K}(\mathrm{t})=\left(\mathrm{K}_{\mathrm{k}}(\mathrm{t})\right)_{\mathrm{k}=1}^{\mathrm{Q}-1}$ is a Skorokhod bounded variation process [4], increasing only when $\phi \in \partial \mathrm{T}_{\mathrm{Q}}(\mathrm{s})$ so as to ensure the boundary condition, eq. (1.3).

Numerical simulations of eq. 1.4 can be efficiently used to make quantitative statements on $\mathcal{P}(\bar{\phi})$, yet they give little insight on its analytic structure. From this point of view, a more convenient approach would be to represent the equilibrium distribution in terms of a properly chosen function basis. A legitimate possibility is to consider polynomial distributions on the Q-simplex. In regard to this choice, we recall that a Dirichlet distribution $\operatorname{Dir}(\gamma)$ of order $\mathrm{Q}$ with parameter $\gamma \in \mathbb{N}^{\mathrm{Q}}$, has probability density

$$
\begin{aligned}
& \mathcal{D}_{\gamma}(\bar{\phi})=\frac{\Gamma(|\gamma|)}{\prod_{k=1}^{\mathrm{Q}} \Gamma\left(\gamma_{\mathrm{k}}\right)} s^{1-|\gamma|}\left(\prod_{k=1}^{\mathrm{Q}-1} \phi_{k}^{\gamma_{k}}\right)(s-|\bar{\phi}|)^{\gamma_{\mathrm{Q}}}, \quad \bar{\phi} \in \overline{\mathrm{T}}_{\mathrm{Q}}(\mathrm{s}), \\
& \overline{\mathrm{T}}_{\mathrm{Q}}(\mathrm{s})=\left\{\bar{\phi} \in \mathbb{R}_{+}^{\mathrm{Q}-1}:|\bar{\phi}| \leqslant s\right\}
\end{aligned}
$$

with $\gamma_{k} \equiv \gamma_{k}-1$ and $|x| \equiv \sum x_{k}$. We notice that $\bar{T}_{Q}(s)$ is in one-to-one correspondence with $\mathrm{T}_{\mathrm{Q}}(\mathrm{s})$, hence we can equivalently write $\phi \in \mathrm{T}_{\mathrm{Q}}(s)$ or $\bar{\phi} \in \overline{\mathrm{T}}_{\mathrm{Q}}(\mathrm{s})$. It is crucial for the reader who is unfamiliar with the mathematics of the simplex to learn how to calculate Dirichlet integrals, i.e. polynomial integrals on $\bar{T}_{Q}(s)$. As an example, we review in App. A an elegant way to work out the normalization constant of eq. 1.5). This is sufficient to be able to reproduce all calculations presented in the paper. That being said, an important feature of the Dirichlet distributions is represented by

Proposition 1. Dirichlet distributions with positive integer indices provide a basis of polynomials, that is to say

$$
\operatorname{span}\left\{\mathcal{D}_{\gamma}(\bar{\phi}): \gamma \in \mathbb{N}^{\mathrm{Q}} \text { and }|\gamma|=\mathrm{n}\right\}=\operatorname{span}\left\{\bar{\phi}^{\alpha}: \alpha \in \mathbb{N}_{0}^{\mathrm{Q}-1} \text { and }|\alpha| \leqslant n\right\}
$$

where we make use of the multi-index notation $\bar{\phi}^{\alpha} \equiv \phi_{1}^{\alpha_{1}} \cdot \ldots \cdot \phi_{\mathrm{Q}-1}^{\alpha_{\mathrm{Q}-1}}$. 
Proof. Given $\gamma \in \mathbb{N}^{\mathrm{Q}}$ with $|\gamma|=n, \mathcal{D}_{\gamma}(\bar{\phi})$ is a polynomial with degree $\mathrm{n}$, hence it can be written as a linear combination of monomials with degree $\leqslant n$. Conversely, suppose that $\alpha \in \mathbb{N}_{0}^{Q-1}$ and $|\alpha|=n$. Then $\bar{\phi}^{\alpha} \propto \mathcal{D}_{\gamma}(\bar{\phi})$ with $\gamma=\left(\alpha_{1}+1, \ldots, \alpha_{\mathrm{Q}-1}+1,1\right)$. Finally, if $\alpha \in \mathbb{N}_{0}^{\mathrm{Q}-1}$ and $|\alpha|=m<n$, then we define $\gamma=\left(\alpha_{1}+1, \ldots, \alpha_{\mathrm{Q}-1}+1, \mathrm{n}-\mathrm{m}+1\right)$ such that $|\gamma|=\mathrm{n}$, and we observe that

$$
\mathcal{D}_{\gamma}(\bar{\phi}) \propto \bar{\phi}^{\alpha}(s-|\bar{\phi}|)^{\mathrm{n}-\mathrm{m}} \propto \bar{\phi}^{\alpha}+\varepsilon_{\alpha}(\bar{\phi})
$$

with $\varepsilon_{\alpha}(\bar{\phi})$ being a linear combination of monomials, each with degree $>m$. Therefore, the proof can be obtained by backward induction on $m=n-1, n-2, \ldots$.

Motivated by this observation, we introduce a polynomial approximation $\mathcal{P}_{n}$ to $\mathcal{P}$ with degree $n$, reading

$$
\begin{aligned}
\mathcal{P}_{\mathrm{n}}(\bar{\phi}) & =\sum_{\gamma \in \Omega_{\mathrm{n}}} \mathrm{c}_{\gamma} \mathcal{D}_{\gamma}(\bar{\phi}), \quad \Omega_{\mathrm{n}}=\left\{\gamma \in \mathbb{N}^{\mathrm{Q}}:|\gamma|=\mathrm{n}\right\}, \\
\sum_{\gamma \in \Omega_{\mathrm{n}}} \mathrm{c}_{\gamma} & =1 .
\end{aligned}
$$

Owing to Prop. 1, eq. 1.9 is equivalent to a complete sum over all monomials of degree $\leqslant n$, while eq. 1.10 is just obtained by imposing that $\mathcal{P}_{n}$ is correctly normalized on $\overline{\mathrm{T}}_{\mathrm{Q}}(\mathrm{s})$. Sometimes, $\Omega_{\mathrm{n}}$ is referred to by mathematicians as the bucket space. The choice of the Dirichlet distributions as a polynomial basis is favourable for several reasons, as we shall explain in next sections.

The aim of the present paper is to describe how estimates of the expansion coefficients $c \equiv\left\{\mathfrak{c}_{\alpha}\right\}$ can be determined straightaway from the FPE, with a view to providing a hopefully helpful analysis tool to practitioners in the physics of complex systems. To this end, we adapt to eqs. (1.2)-(1.3) a mathematical technique known as the Ritz-Galerkin (RG) method for partial differential equations (see for instance [5] for a technical introduction), which is commonly used by engineers in many applicative fields, including fluid and solid mechanics, hydrodynamics, wave propagation, electromagnetism and many others [6]. Our approach makes use of orthogonal polynomials on $\overline{\mathrm{T}}_{\mathrm{Q}}(\mathrm{s})$ as test functions and point-like zero-orthogonal-flux conditions on $\partial \overline{\mathrm{T}}_{\mathrm{Q}}(\mathrm{s})$.

The paper, which is written in a pedagogical style with detailed calculations, is organized as follows. In sect. 2, we provide a short compendium of orthogonal polynomials on $\overline{\mathrm{T}}_{\mathrm{Q}}(\mathrm{s})$, while in sect. 3 we review the basics of the RG method and discuss how to apply it to the FPE for birth-death models with polynomial drift and diffusion coefficients. In sects. 4 and 5, we show applications of the method respectively to the binary voter model with zealots studied in [7], for which an exact solution of the FPE is known, and to its generalization to the multi-state case. In sect. 6, we discuss how symmetry arguments can help reduce the computational budget needed to implement the method. We finally draw our conclusions in sect. 7 .

\section{Orthogonal polynomials on the simplex}

Since $\mathcal{D}_{\gamma}$ is a polynomial distribution on $\bar{T}_{Q}(s)$ with degree $n$ for $\gamma \in \Omega_{n}$, it is rather natural to look for orthogonal polynomial bases on the simplex. The general theory of multivariate orthogonal polynomials is still an open research field: it does not belong to the average undergraduate background of physicists and is not even discussed in many essays in the mathematical literature. Fortunately, an excellent introduction is provided in ref. [8]. We refer the reader to that book for a comprehensive presentation of classical and recent developments on the subject, while for the sake of readability and self-consistency of the paper we review here those aspects which are closely related to our ends. 
First of all, a multivariate polynomial $\mathrm{P}_{\alpha}$ on $\overline{\mathrm{T}}_{\mathrm{Q}}(\mathrm{s})$, indexed by $\alpha \in \mathbb{N}_{\mathrm{Q}}^{\mathrm{Q}-1}$ can be always represented by its monomial expansion

$$
P_{\alpha}(\bar{\phi})=\sum_{\beta \leqslant \alpha} c_{\beta} \bar{\phi}^{\beta} \equiv \sum_{\beta_{1}=0}^{\alpha_{1}} \ldots \sum_{\beta_{\mathrm{Q}-1}=0}^{\alpha_{\mathrm{Q}-1}} c_{\beta_{1} \ldots \beta_{\mathrm{Q}-1}} \phi_{1}^{\beta_{1}} \ldots \phi_{\mathrm{Q}-1}^{\beta_{\mathrm{Q}-1}} .
$$

The degree of $P_{\alpha}$ is the maximum degree of its monomials, i.e. $\operatorname{deg}\left\{P_{\alpha}\right\}=|\alpha|$. Secondly, the orthogonality notion on $\overline{\mathrm{T}}_{\mathrm{Q}}(\mathrm{s})$ depends on the introduction of a scalar product, which in turn requires the specification of a measure. The standard choice - which we adopt here - is to weight the Lebesgue measure by a Dirichlet distribution, i.e. to define

$$
\langle f, g\rangle_{\kappa}=\int_{\bar{T}_{Q}(s)} \mathrm{d} \bar{\phi} f(\bar{\phi}) g(\bar{\phi}) \mathcal{D}_{\kappa}(\bar{\phi}), \quad \kappa \in \mathbb{N}^{\mathrm{Q}},
$$

for sufficiently regular functions $f, g$ on $\bar{T}_{Q}(s)$. The Dirichlet weight is such that $\langle 1,1\rangle_{\kappa}=1$. Thirdly, two polynomials $\mathrm{P}$ and $\mathrm{Q}$ on $\overline{\mathrm{T}}_{\mathrm{Q}}(\mathrm{s})$ are said to be orthogonal if $\langle\mathrm{P}, \mathrm{Q}\rangle_{\kappa}=0$, while a polynomial $\mathrm{P}$ is called an orthogonal polynomial if it is orthogonal to all polynomials of lower degree, i.e.

$$
\langle\mathrm{P}, \mathrm{Q}\rangle_{\mathrm{K}}=0, \quad \forall \mathrm{Q} \quad \text { with } \quad \operatorname{deg}\{\mathrm{Q}\}<\operatorname{deg}\{\mathrm{P}\} .
$$

The main difference between orthogonal polynomial bases in one and several variables, is that the former count just one element per degree, whereas the latter count many of them. To be precise, it can be shown as a trivial consequence of Prop. 1 that

$$
\operatorname{dim}\left\{\text { orthogonal polynomials } \mathrm{P} \text { on } \overline{\mathrm{T}}_{\mathrm{Q}}(\mathrm{s}): \operatorname{deg}\{\mathrm{P}\} \leqslant n\right\}=\left|\Omega_{n}\right|=\left(\begin{array}{c}
n+\mathrm{Q}-1 \\
n
\end{array}\right) .
$$

Notice that the bucket space expands roughly as

$$
\left|\Omega_{\mathrm{n}}\right| \approx \frac{\exp \left\{(\mathrm{Q}-1)\left(\mathrm{H}_{\mathrm{n}}-\gamma_{\mathrm{E}}\right)\right\}}{\Gamma(\mathrm{Q})}, \quad \mathrm{H}_{\mathrm{n}}=\sum_{\mathrm{k}=1}^{\mathrm{n}} \frac{1}{\mathrm{k}},
$$

with $H_{n}$ being the $n$-th harmonic number and $\gamma_{E}=0.57721 \ldots$ the Euler-Mascheroni constant. For this reason, the RG method becomes computationally challenging even for models with a moderately large value of $Q$.

Now, there exist several sets of orthogonal polynomials with respect to eq. [2.2). Along with ref. [8], we focus on two of them, namely

the monomial basis

$$
V_{\alpha}(\bar{\phi})=\sum_{\beta \leqslant \alpha}(-1)^{|\alpha|+|\beta|} s^{-|\beta|} \prod_{i=1}^{\mathrm{Q}-1}\left(\begin{array}{c}
\alpha_{i} \\
\beta_{i}
\end{array}\right) \frac{\left(\kappa_{i}\right)_{\alpha_{i}}}{\left(\kappa_{i}\right)_{\beta_{i}}} \frac{(|\kappa|-1)_{|\alpha|+|\beta|}}{(|\kappa|-1)_{2|\alpha|}} \bar{\phi}^{\beta} \equiv \sum_{\beta \leqslant \alpha} v_{\alpha \beta}(\kappa) \bar{\phi}^{\beta},
$$

the Appel basis

$$
\mathrm{U}_{\alpha}(\bar{\phi})=\mathcal{D}_{\kappa}(\bar{\phi})^{-1} \partial_{\alpha}^{|\alpha|}\left[\phi_{1}^{\alpha_{1}+\kappa_{1}-1} \ldots \phi_{\mathrm{Q}-1}^{\alpha_{\mathrm{Q}-1}+\mathrm{\kappa}_{\mathrm{Q}-1}-1}(s-|\bar{\phi}|)^{|\alpha|+\kappa_{\mathrm{Q}-1}}\right],
$$

for $\alpha \in \mathbb{N}_{0}^{Q-1}$, with $\partial_{\alpha}^{|\alpha|} \equiv \partial^{|\alpha|} / \partial x_{1}^{\alpha_{1}} \ldots \partial x_{d}^{\alpha_{d}}$ and with $(x)_{n}=x(x+1) \ldots(x+n-1)$ denoting the Pochhammer symbol (also known as the raising factorial). The polynomials $\left\{V_{\alpha}\right\}$ and $\left\{U_{\alpha}\right\}$ fulfill the following properties: 
Proposition 2. For any polynomial $\mathrm{P}_{\beta}$ on $\overline{\mathrm{T}}_{\mathrm{Q}}(\mathrm{s})$, it holds

$$
\left\langle\mathrm{V}_{\alpha}, \mathrm{P}_{\beta}\right\rangle_{\kappa}=\left\langle\mathrm{U}_{\alpha}, \mathrm{P}_{\beta},\right\rangle_{\kappa}=0 \text { if }|\beta|<|\alpha| \text {. }
$$

Moreover, the polynomials $\left\{\mathrm{V}_{\alpha}: \alpha \in \mathbb{N}_{0}^{\mathrm{Q}-1}\right\}$ and $\left\{\mathrm{U}_{\alpha}: \alpha \in \mathbb{N}_{0}^{\mathrm{Q}-1}\right\}$ are biorthogonal, i.e. they fulfill

$$
\left\langle\mathrm{V}_{\alpha}, \mathrm{U}_{\beta}\right\rangle_{\mathrm{k}}=\mathrm{f}_{\alpha} \delta_{\alpha \beta}, \quad \mathrm{f}_{\alpha}=\mathrm{s}^{|\alpha|} \frac{\left[\prod_{\mathrm{m}=1}^{\mathrm{Q}-1}\left(\mathrm{k}_{\mathrm{m}}\right)_{\alpha_{\mathrm{m}}} \Gamma\left(\alpha_{\mathrm{m}}+1\right)\right]\left(\mathrm{k}_{\mathrm{Q}}\right)_{|\alpha|}}{(|\mathrm{k}|)_{2|\alpha|}} .
$$

Proof. The proof is contained in ref. [8, chap. 2]. Here, we only review the argument showing that $\left\langle V_{\alpha}, P_{\beta}\right\rangle_{K}=0$, since we shall need a formula which is derived along the proof. Owing to Prop. 1, it is sufficient to prove that $\left\langle V_{\alpha}, X_{\gamma}\right\rangle_{\kappa}=0$ for

$$
\mathrm{X}_{\gamma}(\bar{\phi})=\left[\prod_{\mathrm{m}=1}^{\mathrm{Q}-1} \phi_{\mathrm{m}}^{\gamma_{\mathrm{m}}}\right](s-|\bar{\phi}|)^{\gamma_{\mathrm{Q}}}
$$

with $\gamma \in \mathbb{N}^{\mathrm{Q}}$ and $|\gamma|=|\alpha|-1$. Indeed, it holds

$$
\begin{aligned}
& \left\langle\mathrm{V}_{\alpha}, \mathrm{X}_{\gamma}\right\rangle_{\kappa}=\int_{\overline{\mathrm{T}}_{\mathrm{Q}}(s)} \mathrm{d} \bar{\phi} \mathrm{V}_{\alpha}(\bar{\phi}) \mathrm{X}_{\gamma}(\bar{\phi}) \mathcal{D}_{\kappa}(\bar{\phi}) \\
& =\frac{\Gamma(|\kappa|)}{\prod_{k=1}^{\mathrm{Q}} \Gamma\left(\kappa_{k}\right)} s^{1-|\kappa|} \sum_{\beta \leqslant \alpha}(-1)^{|\alpha|+|\beta|} s^{-|\beta|} \prod_{m=1}^{Q-1}\left(\begin{array}{l}
\alpha_{m} \\
\beta_{m}
\end{array}\right) \frac{\left(\kappa_{m}\right)_{\alpha_{m}}}{(|\kappa|-1)_{|\alpha|+|\beta|}} \\
& \cdot \int_{\overline{\mathrm{T}}_{\mathrm{Q}}(s)} \mathrm{d} \bar{\phi} \prod_{\ell=1}^{\mathrm{Q}-1} \phi_{\ell}^{\beta_{\ell}+\gamma_{\ell}+\kappa_{\ell}-1}(s-|\bar{\phi}|)^{\mathrm{K}_{\mathrm{Q}}+\gamma_{\mathrm{Q}}-1} \\
& =s^{|\chi|} \frac{\Gamma(|\kappa|)}{\Gamma(|\kappa|+2|\alpha|-1)}\left[\prod_{m=1}^{\mathrm{Q}-1} \frac{\Gamma\left(\kappa_{m}+\alpha_{m}\right) \Gamma\left(\gamma_{m}+1\right)}{\Gamma\left(\kappa_{m}\right)}\right] \frac{\Gamma\left(\kappa_{Q}+\gamma_{Q}\right)}{\Gamma\left(\kappa_{Q}\right)} \\
& \cdot \sum_{\beta \leqslant \alpha} \prod_{m=1}^{Q-1}(-1)^{\beta_{m}}\left(\begin{array}{c}
\alpha_{m} \\
\beta_{m}
\end{array}\right)\left(\begin{array}{c}
\kappa_{m}+\gamma_{m}+\beta_{m}-1 \\
\gamma_{m}
\end{array}\right) \frac{\Gamma(|\kappa|+|\alpha|+|\beta|-1)}{\Gamma(|\kappa|+|\gamma|+|\beta|)} .
\end{aligned}
$$

If $|\gamma|=|\alpha|-1$, the rightmost ratio of $\Gamma$-functions simplifies and we are left with a product of independent sums. From a Chu-Vandermonde formula

$$
\sum_{k=0}^{n}(-1)^{k}\left(\begin{array}{l}
n \\
k
\end{array}\right)\left(\begin{array}{c}
a+k \\
m
\end{array}\right)=\frac{(-m)_{n}}{\Gamma(m+1)(1+a)_{n-m}}, \quad m, n \in \mathbb{N}, a \in \mathbb{R},
$$

it follows

$$
\begin{aligned}
\left\langle\mathrm{V}_{\alpha}, \mathrm{X}_{\gamma}\right\rangle_{\mathrm{k}} & =\mathrm{s}^{|\gamma|} \frac{\Gamma(|\mathrm{k}|)}{\Gamma(|\mathrm{k}|+2|\alpha|-1)}\left[\prod_{\mathrm{m}=1}^{\mathrm{Q}-1} \frac{\Gamma\left(\mathrm{k}_{\mathrm{m}}+\alpha_{\mathrm{m}}\right) \Gamma\left(\gamma_{\mathrm{m}}+1\right)}{\Gamma\left(\kappa_{\mathrm{m}}\right)}\right] \frac{\Gamma\left(\mathrm{k}_{\mathrm{Q}}+\gamma_{\mathrm{Q}}\right)}{\Gamma\left(\kappa_{\mathrm{Q}}\right)} \\
& \cdot \prod_{\mathrm{m}=1}^{\mathrm{Q}-1} \frac{\left(-\gamma_{\mathrm{m}}\right)_{\alpha_{\mathrm{m}}}}{\Gamma\left(\gamma_{\mathrm{m}}+1\right)\left(\mathrm{k}_{\mathrm{m}}+\gamma_{\mathrm{m}}\right)_{\alpha_{\mathrm{m}}-\gamma_{\mathrm{m}}}} .
\end{aligned}
$$

However, we know that $(-m)_{n}=0$ for $m<n$. Since $|\gamma|=|\alpha|-1$, there is at least one value of $m$ for which $\gamma_{m}<\alpha_{m}$. Therefore we conclude that $V_{\alpha}$ is orthogonal to $X_{\gamma}$.

Remark 1. The polynomials $\left\{\mathrm{V}_{\alpha}\right\}$ can be easily coded. Indeed, we use them for computations. However, it should be noticed that the numerical evaluation of eq. (2.6) can be critical, specially for $|\alpha| \gg 1$, since $V_{\alpha}$ adds largely different ratios of factorials with alternating signs. For this reason, computations should be performed and crosschecked with different levels of floating point rounding. Most of the numerical experiments described in next sections have been done in Maple ${ }^{T M}$, which allows to control the numerical precision by the environment variable Digits. We employ the biorthogonal basis $\left\{\mathrm{U}_{\alpha}\right\}$ essentially to develop theoretical arguments. 


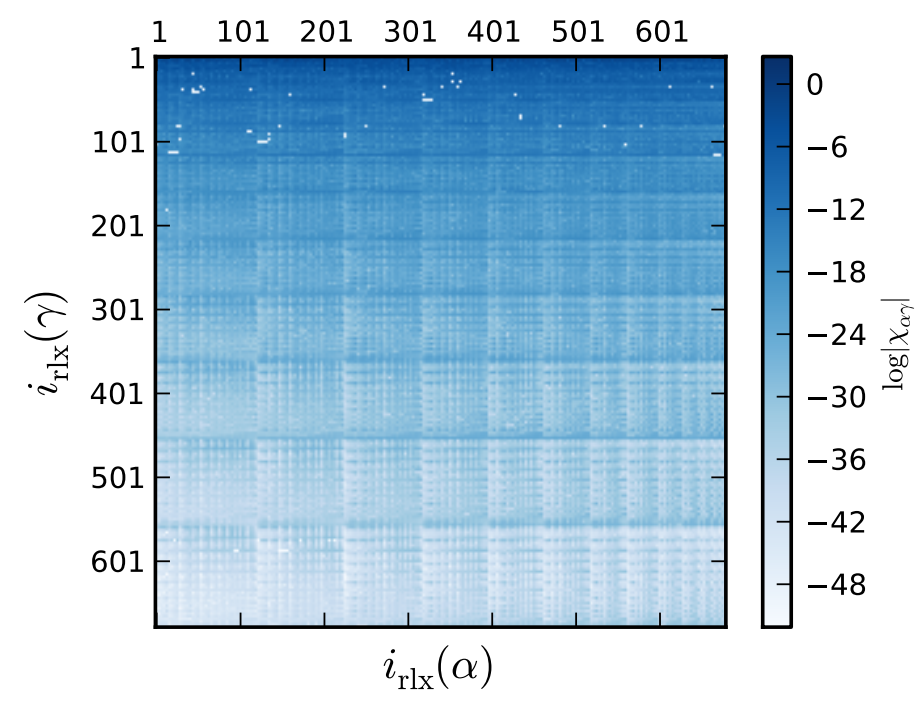

Figure 1 - Heat map of $\log \left|\chi_{\alpha \gamma}\right|$ for $Q=4, n=14, k=(2,2,2,2)$ and $s=1$. In this case $\left|\Omega_{n}\right|=680$.

If $|\gamma| \neq|\alpha|-1$, the factorization property does not hold, hence we are left with the general formula

$$
\begin{aligned}
\chi_{\alpha \gamma} \equiv\left\langle\mathrm{V}_{\alpha}, \mathcal{D}_{\gamma}\right\rangle_{\kappa}=s^{1-\mathrm{Q}} \frac{\Gamma(|\gamma|) \Gamma(|\kappa|)}{\Gamma(|\mathrm{k}|+2|\alpha|-1)}\left[\prod_{m=1}^{\mathrm{Q}-1} \frac{\Gamma\left(\kappa_{\mathrm{m}}+\alpha_{\mathrm{m}}\right)}{\Gamma\left(\kappa_{\mathrm{m}}\right)}\right] \frac{\Gamma\left(\kappa_{\mathrm{Q}}+\gamma_{\mathrm{Q}}-1\right)}{\Gamma\left(\kappa_{\mathrm{Q}}\right) \Gamma\left(\gamma_{\mathrm{Q}}\right)} \\
\cdot \sum_{\beta \leqslant \alpha} \frac{\Gamma(|\mathrm{k}|+|\alpha|+|\beta|-1)}{\Gamma(|\mathrm{k}|+|\gamma|+|\beta|-\mathrm{Q})} \prod_{\mathrm{m}=1}^{\mathrm{Q}-1}(-1)^{\beta_{\mathrm{m}}}\left(\begin{array}{c}
\alpha_{\mathrm{m}} \\
\beta_{\mathrm{m}}
\end{array}\right)\left(\begin{array}{c}
\kappa_{\mathrm{m}}+\gamma_{\mathrm{m}}+\beta_{\mathrm{m}}-2 \\
\gamma_{\mathrm{m}}-1
\end{array}\right),
\end{aligned}
$$

valid for $|\alpha| \leqslant|\gamma|$. We can extend Remark 1 to eq. 2.14 as well. The meaning of the matrix $\chi=\left\{\chi_{\alpha \gamma}\right\}$ becomes clear if we expand $\mathcal{D}_{\gamma}$ along the Appel basis, namely

$$
\mathcal{D}_{\gamma}(\bar{\phi})=\sum_{0 \leqslant|\beta| \leqslant|\gamma|} d_{\gamma \beta} \mathrm{U}_{\beta}(\bar{\phi}) .
$$

By projecting both sides of eq. (2.15) onto $\mathrm{V}_{\alpha}$, we obtain

$$
\chi_{\alpha \gamma}=\left\langle V_{\alpha}, \mathcal{D}_{\gamma}\right\rangle_{\kappa}=\sum_{0 \leqslant|\beta| \leqslant|\gamma|} d_{\gamma \beta}\left\langle V_{\alpha}, U_{\beta}\right\rangle_{\kappa}=\sum_{0 \leqslant|\beta| \leqslant|\gamma|} d_{\gamma \beta} f_{\beta} \delta_{\alpha \beta}=d_{\gamma \alpha} f_{\alpha}
$$

whence it follows

$$
\mathcal{D}_{\gamma}(\bar{\phi})=\sum_{0 \leqslant|\beta| \leqslant|\gamma|} \frac{\chi_{\beta \gamma}}{f_{\beta}} \mathrm{U}_{\beta}(\bar{\phi}) .
$$

We thus conclude that, given $n \geqslant 0, \gamma \in \Omega_{n}$ and $\alpha \in \mathbb{N}_{0}^{\mathrm{Q}-1}$ such that $|\alpha| \leqslant|\gamma|$, the matrix elements $\chi_{\alpha \gamma}$ are essentially the expansion coefficients of the (non-orthogonal) basis $\left\{\mathcal{D}_{\gamma}\right\}$ along the (orthogonal) Appel basis $\left\{\mathrm{U}_{\alpha}\right\}$, i.e. $\chi$ is essentially a change-of-basis matrix. It is interesting to look at the numerical values of $\chi_{\alpha \gamma}$ in some specific case. As an example, in Fig. 1 we show a heat map of $\log \left|\chi_{\alpha \gamma}\right|$ for $Q=4$, $n=14, k=(2,2,2,2)$ and $s=1$; here, the index arrays are sorted in their respective domains according to a reverse lexicographic ordering (RLO) $\alpha \rightarrow i_{\text {rlx }}(\alpha)$, which we recall to be defined by

Definition 1. Given $d \geqslant 1$ and $\alpha, \beta \in \mathbb{N}_{0}^{\mathrm{d}}$, we say that $\alpha \prec \beta$ if $|\alpha|<|\beta|$ or $|\alpha|=|\beta|$ and $\exists k \in\{1, \ldots, \mathrm{d}\}$ : $\alpha_{i}=\beta_{i}$ for $i=1 \ldots k-1$, and $\alpha_{k}<\beta_{k}$. 
An efficient indexing algorithm for this specific ordering is discussed in [9], to which we refer the reader for details. We see from the heat map that the coefficients $\chi_{\alpha \gamma}$ decrease exponentially as $|\alpha|$ increases. This behaviour looks natural if one considers that $V_{\alpha}$ is not positive definite on $\bar{T}_{Q}(s)$ : its zeros are an algebraic variety, whose structure becomes more and more complex as $|\alpha|$ increases. The sign of $V_{\alpha}$ is important since $\mathcal{D}_{\gamma}(\bar{\phi}) \geqslant 0$ for $\bar{\phi} \in \bar{T}_{Q}(s)$, hence $\chi_{\alpha \gamma}$ receives contributions of opposite signs from adjacent domains separated by zeros of $V_{\alpha}$. For this reason, it averages progressively to zero as $|\alpha|$ increases. The isolated white points in the upper part of the plot correspond to values of $\alpha$ and $\gamma$ for which $\chi_{\alpha \gamma}=0$; they have been coloured as the lowest non-zero observed value of $\log \left|\chi_{\alpha \gamma}\right|$, just to preserve the colour map.

The reader could feel uncomfortable with the fact that the orthogonal bases are not normalized on $\bar{T}_{Q}(s)$ such as $\mathcal{D}_{\gamma}$ is. This is not really a problem as far as we are concerned, since normalization constants change the rows of the RG matrix by an irrelevant overall rescaling, as we shall see in the next section. Moreover, normalizing the orthogonal polynomials $\left\{\mathrm{V}_{\alpha}\right\}$ and $\left\{\mathrm{U}_{\alpha}\right\}$ as if they were probability densities on $\bar{T}_{Q}(s)$ is not possible. Indeed, their integrals $Z_{V}$ and $Z_{U}$, which are discussed in App. A for the sake of completeness, vanish for specific values of $\alpha$ and $k$ due to non-positiveness.

\section{Ritz-Galerkin orthogonality}

The Fokker-Planck operator $\mathcal{L}_{\mathrm{FP}}$ is a linear operator: given two functions $\mathcal{P}_{1}, \mathcal{P}_{2} \in \mathcal{H}$, with $\mathcal{H}$ a sufficiently regular function space on the $\mathrm{Q}$-simplex, such that $\mathcal{L}_{\mathrm{FP}} \cdot \mathcal{P}_{\mathrm{k}}=0$ for $\mathrm{k}=1,2$, then $\mathcal{L}_{\mathrm{FP}}$. $\left(a_{1} \mathcal{P}_{1}+a_{2} \mathcal{P}_{2}\right)=0$ for any $a_{1}, a_{2} \in \mathbb{R}$. In other words, the solutions of the stationary FPE belong to $\operatorname{ker} \mathcal{L}_{\mathrm{FP}} \subset \mathcal{H}$. Since in general $\operatorname{dim} \operatorname{ker} \mathcal{L}_{\mathrm{FP}}>1$, a specific solution can be singled out by imposing a set of additional conditions. We shall come to this point in a while. For the time being, we observe that

$$
\left\langle\mathrm{V}, \mathcal{L}_{\mathrm{FP}} \cdot \mathcal{P}\right\rangle_{\mathrm{K}}=0 \quad \forall \mathrm{V} \in \mathcal{H} \quad \text { if } \quad \mathcal{P} \in \operatorname{ker} \mathcal{L}_{\mathrm{FP}} .
$$

Conversely, a function $\mathcal{P} \in \mathcal{H}$ fulfilling $\left\langle\mathrm{V}, \mathcal{L}_{\mathrm{FP}} \cdot \mathcal{P}\right\rangle_{\mathrm{K}}=0 \quad \forall \mathrm{V} \in \mathcal{H}$ is called a weak solution of the FPE. The idea underlying the RG approximation method is to look for a weak solution by enforcing eq. (3.1) only for $V \in \overline{\mathcal{H}}$, with $\overline{\mathcal{H}}$ a properly chosen subset of $\mathcal{H}$. For instance, for $n \geqslant 1$, we could opt for

$$
\overline{\mathcal{H}}_{\mathrm{n}}=\operatorname{span}\left\{\bar{\phi}^{\alpha}: \alpha \in \mathbb{N}_{\mathrm{O}}^{\mathrm{Q}-1} \text { and }|\alpha| \leqslant n\right\} .
$$

Since $\left\{\mathrm{V}_{\alpha}\right\}_{|\alpha| \leqslant n}$ is a basis of $\overline{\mathcal{H}}_{n}$, a RG weak solution $\mathcal{P}_{n}$ has to fulfill

$$
\left\langle\mathrm{V}_{\alpha}, \mathcal{L}_{\mathrm{FP}} \cdot \mathcal{P}_{\mathrm{n}}\right\rangle_{\mathrm{K}}=0 \quad \forall \alpha:|\alpha| \leqslant n .
$$

As proved in a celebrated theorem by Lax and Milgram [10], a sufficient condition to make the search of weak solutions (and therefore of RG weak solutions) a well-posed problem, is that the following two properties are fulfilled:

$$
\begin{array}{lll}
\text { - boundedness } & \rightsquigarrow \mathrm{C}<+\infty: \quad\left|\left\langle\mathrm{V}, \mathcal{L}_{\mathrm{FP}} \cdot \mathrm{V}^{\prime}\right\rangle\right| \leqslant \mathrm{C}\left\|\mathrm { V } \left|\left\|\mid \mathrm{V}^{\prime}\right\|, \quad \forall \mathrm{V}, \mathrm{V}^{\prime} \in \mathcal{H},\right.\right. \\
\text { - coerciveness } & \rightsquigarrow \quad \exists \mathrm{c}>0: \quad\left|\left\langle\mathrm{V}, \mathcal{L}_{\mathrm{FP}} \cdot \mathrm{V}\right\rangle\right| \geqslant \mathrm{c}\|\mathrm{V}\|^{2}, \quad \forall \mathrm{V} \in \mathcal{H},
\end{array}
$$

for some scalar product $\langle\cdot, \cdot\rangle$ on $\overline{\mathrm{T}}_{\mathrm{Q}}(\mathrm{s})$ (not necessarily $\langle\cdot, \cdot\rangle_{\mathrm{K}}$ ), with $\|\cdot\|^{2}=\langle\cdot, \cdot\rangle$ being the induced norm. Though it is not difficult to check the boundedness condition for a $\mathcal{L}_{F P}$ with polynomial coefficients $\left(A_{k}\right)_{k=1}^{Q-1}$ and $\left(B_{i k}\right)_{i, k=1}^{Q-1}$ on a compact domain such as $\bar{T}_{Q}(s)$, checking the coerciveness of $\mathcal{L}_{F P}$ is more problematic, since this is related to the structure of the eigenvalue spectrum of $\left(B_{i k}\right)_{i, k=1}^{Q-1}$. We do not attempt any general proof in the present paper. Instead, we adopt a heuristic approach where 
we just apply the RG method to a given complex model and check out the outcome. However, if the Lax-Milgram conditions are fulfilled, then the Céa estimate

$$
\left\|\mathcal{P}-\mathcal{P}_{n}\right\| \leqslant \frac{C}{c} \inf _{Q_{n} \in \mathcal{H}_{n}}\left\|\mathcal{P}-Q_{n}\right\|
$$

follows straightaway, stating that the RG solution $\mathcal{P}_{\mathrm{n}}$ is a quasi-best approximation on $\overline{\mathcal{H}}_{\mathrm{n}}$ to a truly weak solution $\mathcal{P} \in \mathcal{H}$. In addition, the error $\mathcal{P}-\mathcal{P}_{n}$ is weakly orthogonal to $\overline{\mathcal{H}}_{n}$. That being said, we are ready to show how to adapt the RG method to birth-death models with polynomial drift and diffusion coefficients.

i) If we expand $\mathcal{P}_{n}$ according to eq. (1.9) and insert the expansion into eq. 3.3, we obtain

$$
0=\sum_{\gamma \in \Omega_{n}}\left\langle\mathrm{~V}_{\alpha}, \mathcal{L}_{\mathrm{FP}} \cdot \mathcal{D}_{\gamma}\right\rangle_{\mathrm{K}} \mathrm{c}_{\gamma}=\sum_{\gamma \in \Omega_{\mathrm{n}}} \psi_{\alpha \gamma} \mathrm{c}_{\gamma}, \quad \psi_{\alpha \gamma} \equiv\left\langle\mathrm{V}_{\alpha}, \mathcal{L}_{\mathrm{FP}} \cdot \mathcal{D}_{\gamma}\right\rangle_{\kappa} .
$$

Accordingly, the stationary FPE turns into a square homogeneous linear system with coefficient matrix $\psi \in \mathbb{R}^{\left|\Omega_{n}\right| \times\left|\Omega_{n}\right|}$ and unknown vector $c \in \mathbb{R}^{\left|\Omega_{n}\right|}$, both indexed (for instance) via $\alpha \rightarrow i_{\text {rlx }}(\alpha)$. If the problem is well posed, the eigenvalue spectrum of $\psi$ must have a certain number of zeros $v_{0}>0$, depending on $n, Q$ and the specific form of $\left(A_{k}\right)_{k=1}^{Q-1}$ and $\left(B_{i k}\right)_{i, k=1}^{Q-1}$. The linear system $\psi \cdot c=0$ must be augmented by imposing that $c$ is normalized according to eq. 1.10 and by introducing a set of - say $-\mathrm{Qn}_{\mathrm{bc}}$ additional equations to enforce the boundary conditions, eq. 1.3. This leads us to a larger non-homogeneous linear system $\Psi \cdot c=\eta$, of which we know at present that

$$
\begin{array}{ll}
\Psi \in \mathbb{R}^{\left(\left|\Omega_{n}\right|+1+Q n_{b c}\right) \times\left|\Omega_{n}\right|}: \begin{cases}\Psi_{i j}=\psi_{i j} & \text { for } i, j=1, . .,\left|\Omega_{n}\right| ; \\
\Psi_{\left(\left|\Omega_{n}\right|+1\right) j}=1 & \text { for } j=1, \ldots,\left|\Omega_{n}\right| ;\end{cases} \\
\eta \in \mathbb{R}^{\left(\left|\Omega_{n}\right|+1+Q n_{b c}\right)}: & \begin{cases}\eta_{i}=0 & \text { for } i=1, . .,\left|\Omega_{n}\right| ; \\
\eta_{\left|\Omega_{n}\right|+1}=1 .\end{cases}
\end{array}
$$

It should be observed that eq. 3.7] is left invariant by any change of normalization of $V_{\alpha}$. Such a change would just correspond to rescaling the rows of $\psi$.

ii) Let us see how to set up the boundary conditions and fill in the lowest $\mathrm{Qn}_{\mathrm{bc}}$ rows of $\Psi$ and elements of $\eta$. First, we recall that the stationary FPE can be written in the form of a local conservation law, namely

$$
0=\sum_{k=1}^{\mathrm{Q}-1} \partial_{k} \mathrm{~J}_{k}(\bar{\phi}), \quad \mathrm{J}_{k}(\bar{\phi})=-A_{k}(\bar{\phi}) \mathcal{P}(\bar{\phi})+\frac{1}{2} \sum_{i=1}^{\mathrm{Q}-1} \partial_{i}\left[\mathrm{~B}_{i k}(\bar{\phi}) \mathcal{P}(\bar{\phi})\right],
$$

where $J$ is naturally interpreted as a vector probability current. Integrating both sides of eq. (3.10) over $\overline{\mathrm{T}}_{\mathrm{Q}}(\mathrm{s})$ and making use of the divergence theorem yields

$$
0=\int_{\partial \overline{\mathrm{T}}_{\mathrm{Q}}(\mathrm{s})} \mathrm{d} \bar{\phi} \hat{\mathrm{n}}(\bar{\phi}) \cdot J(\bar{\phi}), \quad \mathrm{Q}>2,
$$

with $\hat{n}(\bar{\phi})$ representing the inward pointing unit vector orthogonal to $\partial \overline{\mathrm{T}}_{\mathrm{Q}}(\mathrm{s})$ at $\bar{\phi}$. Clearly, eq. 3.11) means that there is no overall probability flux across $\partial \bar{T}_{Q}(s)$ when the system is in equilibrium. In order for eq. (1.3) to hold, the orthogonal component of the probability current must vanish point-bypoint on the boundary (reflecting boundary conditions) and not just on average, i.e. the sought solution must fulfill $\hat{n}(\bar{\phi}) \cdot J(\bar{\phi})=0$ for $\bar{\phi} \in \partial \overline{\mathrm{T}}_{\mathrm{Q}}(\mathrm{s})$. Unfortunately, this is a continuous infinite set of conditions, 


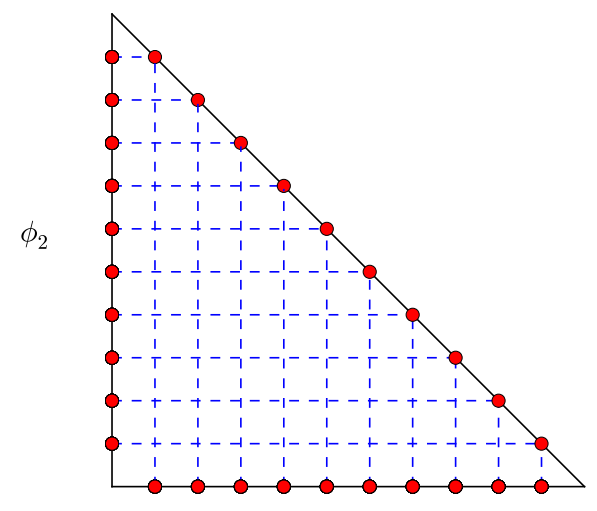

$\phi_{1}$

Figure 2 - Zero-orthogonal-flux points for $\mathrm{Q}=3$ and $\mathrm{n}_{\mathrm{bc}}=10$.

which we however approximate by a finite subset. To this end, we observe that $\partial \overline{\mathrm{T}}_{\mathrm{Q}}(\mathrm{s})$ is made of $Q$ ( $\mathrm{Q}-1)$-dimensional hypersurfaces, namely

$$
\partial \overline{\mathrm{T}}_{\mathrm{Q}}(\mathrm{s})=\bigcup_{\mathrm{k}=1}^{\mathrm{Q}} \mathfrak{H}_{\mathrm{k}}, \quad \begin{cases}\mathfrak{H}_{\mathrm{k}}=\left\{\bar{\phi} \in \overline{\mathrm{T}}_{\mathrm{Q}}(\mathrm{s}):\right. & \left.\phi_{\mathrm{k}}=0\right\} ; \quad \mathrm{k}=1, \ldots, \mathrm{Q}-1, \\ \mathfrak{H}_{\mathrm{Q}}=\left\{\bar{\phi} \in \overline{\mathrm{T}}_{\mathrm{Q}}(\mathrm{s}):\right. & |\bar{\phi}|=s\} .\end{cases}
$$

On each hypersurface $\mathfrak{H}_{k}$ we consider a regular grid of zero-orthogonal-flux points $\left(\bar{\phi}_{k m}\right)_{m=1}^{n_{b c}}$ at which we impose the condition $0=\hat{n}\left(\bar{\phi}_{\mathrm{km}}\right) \cdot J\left(\bar{\phi}_{\mathrm{km}}\right)$, i.e.

$$
\begin{array}{ll}
0=J_{k}\left(\bar{\phi}_{k m}\right), & k=1, \ldots, Q-1 \quad m=1, \ldots, n_{b c}, \\
0=\sum_{k=1}^{Q-1} J_{k}\left(\bar{\phi}_{\mathrm{Qm}}\right), & m=1, \ldots, n_{b c} .
\end{array}
$$

An illustrative example corresponding to $Q=3$ and $n_{b c}=10$ is shown in Fig. 2, where the boundary points have been chosen according to

$$
\bar{\phi}_{1 \mathrm{~m}}=\left(0, s \frac{\mathrm{m}}{n_{\mathrm{bc}}+1}\right), \quad \bar{\phi}_{2 \mathrm{~m}}=\left(s \frac{\mathrm{m}}{n_{\mathrm{bc}}+1}, 0\right), \quad \bar{\phi}_{3 \mathrm{~m}}=\left(s \frac{\mathrm{m}}{\mathrm{n}_{\mathrm{bc}}+1}, s \frac{n_{\mathrm{bc}}+1-\mathrm{m}}{n_{\mathrm{bc}}+1}\right) .
$$

iii) As already observed, the rank $r_{\psi}$ of $\psi$ is expected not to be maximal, i.e. $r_{\psi}=\left|\Omega_{n}\right|-v_{0}<\left|\Omega_{n}\right| \equiv$ $r_{\psi, \max }$. The normalization condition eq. 1.10 adds a linearly independent row to the system $\psi \cdot c=0$, thus increasing the rank of the coefficient matrix by one. Each additional boundary condition adds another linearly independent row and further increases the rank of the coefficient matrix until this becomes maximal. From this point on, i.e. for $\mathrm{Qn}_{\mathrm{bc}}>v_{0}-1$, the rank of the coefficient matrix keeps maximal, while the system becomes overconstrained and thus inconsistent (to understand this, imagine to perform a row echelon reduction of the system $\Psi \cdot c=\eta \rightarrow \Psi_{\text {red }} \cdot c=\eta_{\text {red }}$; the reduced row echelon form $\Psi_{\text {red }}$ has still maximal rank $r_{\Psi}=\left|\Omega_{n}\right|$; its last $\mathrm{Qn}_{\mathrm{bc}}-v_{0}+1$ rows are full of zeros, while in general the last $Q n_{\mathrm{bc}}-v_{0}+1$ elements of $\eta_{\text {red }}$ are expected not to vanish). This is particularly inconvenient, as it compels us to very carefully choose an exact number $\mathrm{Q} n_{\mathrm{bc}}=v_{0}-1$ of boundary points. Though reasonable, we have no theoretical argument to prove that $v_{0}-1 \propto \mathrm{Q}$. We follow a different approach: 
an alternative is indeed to impose an arbitrary number $Q n_{b c}>v_{0}-1$ of boundary conditions and consider the normal system

$$
\left(\Psi^{\mathrm{T}} \cdot \Psi\right) \cdot c=\Psi^{\mathrm{T}} \cdot \eta \text {. }
$$

in place of the original one (least-squares problem). Eq. (3.16) is consistent for any choice of $\mathrm{Q} n_{\mathrm{bc}}>$ $v_{0}-1$. Indeed, since $\Psi$ has maximal rank, $\Psi^{\mathrm{T}} \cdot \Psi \in \mathbb{R}^{\left|\Omega_{n}\right| \times\left|\Omega_{n}\right|}$ has no zero eigenvalues, hence it can be inverted. The system is consistent as $\left(\Psi^{\mathrm{T}} \cdot \eta\right)_{k}=1$ for all $\mathrm{k}=1, \ldots,\left|\Omega_{\mathrm{n}}\right|+1+\mathrm{Q} \mathrm{n}_{\mathrm{bc}}$, as a consequence of eqs. (3.8)-(3.9). Clearly, the original system and eq. 3.16) are not equivalent.

Notice that $\Psi$ is in general expected to have a large condition number (the ratio between its largest and lowest singular value) and the latter is expected to get larger as $n$ increases. Since the condition number of $\Psi^{\mathrm{T}} \cdot \Psi$ is the square of the condition number of $\Psi$, the inversion of $\Psi^{\mathrm{T}} \cdot \Psi$ might be computationally critical. Therefore, an appropriate inversion algorithm should be used in order to solve eq. (3.16). We use the CGNR algorithm in our numerical tests, see ref. [11, chapt. 8] for details.

The effects of imposing more and more boundary conditions will be discussed in a specific example in sect. 5. We can say in advance that the condition number of $\Psi^{\mathrm{T}} \cdot \Psi$ is not sensitive to $n_{\mathrm{bc}}$ and that $\mathcal{P}_{n}$ rapidly converges as $n_{b c}$ increases.

$i v)$ We need to discuss how to concretely work out and compute the matrix elements $\psi_{\alpha \gamma}$. Here, the assumption that $\left(A_{k}\right)_{k=1}^{Q-1}$ and $\left(B_{i k}\right)_{i, k=1}^{Q-1}$ are polynomials becomes practically decisive. Indeed, we observe that $\partial_{k}^{m} \mathcal{D}_{\gamma}$ is a polynomial on $\bar{T}_{Q}(s)$ with $\operatorname{deg}\left\{\partial_{k}^{m} \mathcal{D}_{\gamma}\right\}=|\gamma|-m$ for $m \leqslant|\gamma|$, while $\phi_{k}^{m} \mathcal{D}_{\gamma}$ is a polynomial on $\bar{T}_{\mathrm{Q}}(\mathrm{s})$ with $\operatorname{deg}\left\{\phi_{\mathrm{k}}^{\mathrm{m}} \mathcal{D}_{\gamma}\right\}=|\gamma|+\mathrm{m}$. Since $\left\{\mathcal{D}_{\gamma}\right\}$ is a polynomial basis, it must be possible to express both $\partial_{\mathrm{k}}^{\mathrm{m}} \mathcal{D}_{\gamma}$ and $\phi_{\mathrm{k}}^{\mathrm{m}} \mathcal{D}_{\gamma}$ as linear combinations of some $\left\{\mathcal{D}_{\gamma^{\prime}}\right\}$. Now, since $\mathcal{L}_{\mathrm{FP}}$ is a second order partial differential operator, we never need to differentiate more than twice. Analogously, since $\mathcal{L}_{\mathrm{FP}}$ is usually derived from a Master Equation resulting from a detailed balance, $\left(A_{k}\right)_{k=1}^{Q-1}$ and $\left(\mathrm{B}_{i k}\right)_{i, k=1}^{\mathrm{Q}-1}$ are usually not more than quadratic polynomials (this statement is of course less universal as the reader may understand - since transition rates depend on the specific model, but is often true). Instead of writing a general formula to expand $\phi_{i}^{p} \phi_{k}^{\mathrm{q}} \partial_{m}^{\mathrm{r}} \partial_{\mathrm{n}}^{s} \mathcal{D}_{\gamma}(\bar{\phi})$ as a linear combination of Dirichlet distributions, we prefer to report formulae for specific choices of indices and exponents. To this aim, we need to introduce some additional notation. We define

$$
\gamma_{\ell^{ \pm}} \equiv\left(\gamma_{1}, \ldots, \gamma_{\ell-1}, \gamma_{\ell} \pm 1, \gamma_{\ell+1}, \ldots, \gamma_{\mathrm{Q}}\right) .
$$

Similarly, we define $\gamma_{\ell^{+} \mathrm{m}^{+}}, \gamma_{\ell^{+} \mathrm{m}^{-}}, \gamma_{\ell^{++}}$, etc. as results of the iterated application of index-raising operators $\oplus_{\ell} \cdot \gamma \equiv \gamma_{\ell^{+}}$and index-lowering operators $\theta_{\ell} \cdot \gamma \equiv \gamma_{\ell^{-}}$, somewhat similar to the creation and destruction operators of the quantum harmonic oscillator. Based on this, reference formulae read

$$
\begin{aligned}
& \phi_{\ell} \mathcal{D}_{\gamma}(\bar{\phi})=s \frac{\gamma_{\ell}}{|\gamma|} \mathcal{D}_{\gamma_{\ell}+}(\bar{\phi}) \\
& \partial_{\ell} \mathcal{D}_{\gamma}(\bar{\phi})=s^{-1}(|\gamma|-1)\left[\theta_{\gamma_{\ell}, 2} \mathcal{D}_{\gamma_{\ell-}}(\bar{\phi})-\theta_{\gamma_{Q}, 2} \mathcal{D}_{\gamma_{Q^{-}}}(\bar{\phi})\right] \\
& \phi_{\ell} \partial_{\ell} \mathcal{D}_{\gamma}(\bar{\phi})=\theta_{\gamma_{\ell}, 2}\left(\gamma_{\ell}-1\right) \mathcal{D}_{\gamma}(\bar{\phi})-\theta_{\gamma_{Q}, 2} \gamma_{\ell} \mathcal{D}_{\gamma_{\ell+Q^{-}}}(\bar{\phi}), \\
& \phi_{\ell}^{2} \partial_{\ell} \mathcal{D}_{\gamma}(\bar{\phi})=\frac{s}{|\gamma|}\left\{\theta_{\gamma_{\ell}, 2 \gamma_{\ell}}\left(\gamma_{\ell}-1\right) \mathcal{D}_{\gamma_{\ell+}}(\bar{\phi})-\theta_{\gamma_{Q}, 2} \gamma_{\ell}\left(\gamma_{\ell}+1\right) \mathcal{D}_{\gamma_{\ell++} Q^{-}}(\bar{\phi})\right\}
\end{aligned}
$$




$$
\begin{gathered}
\left.\phi_{\ell} \partial_{\mathrm{m}} \mathcal{D}_{\gamma}(\bar{\phi})\right|_{\ell \neq \mathrm{m}}=\gamma_{\ell}\left[\theta_{\gamma_{\mathrm{m}}, 2} \mathcal{D}_{\gamma_{\ell^{+} \mathrm{m}}}(\bar{\phi})-\theta_{\gamma_{\mathrm{Q}}, 2} \mathcal{D}_{\gamma_{\ell^{+} \mathrm{Q}^{-}}}(\bar{\phi})\right] \\
\partial_{\ell}^{2} \mathcal{D}_{\gamma}(\bar{\phi})=\mathrm{s}^{-2}(|\gamma|-1)(|\gamma|-2) \cdot\left\{\theta_{\gamma_{\ell}, 3} \mathcal{D}_{\gamma_{\ell^{--}}}(\bar{\phi})\right. \\
\left.-2 \theta_{\gamma_{\ell}, 2} \theta_{\gamma_{\mathrm{Q}}, 2} \mathcal{D}_{\gamma_{\ell-\mathrm{Q}^{-}}}(\bar{\phi})+\theta_{\gamma_{\mathrm{Q}}, 3} \mathcal{D}_{\gamma_{\mathrm{Q}^{--}}}(\bar{\phi})\right\} \\
\phi_{\ell} \partial_{\ell}^{2} \mathcal{D}_{\gamma}(\phi)=\mathrm{s}^{-1}(|\gamma|-1) \cdot\left\{\left(\gamma_{\ell}-2\right) \theta_{\gamma_{\ell}, 3} \mathcal{D}_{\gamma_{\gamma^{-}}}(\bar{\phi})\right. \\
\left.-2\left(\gamma_{\ell}-1\right) \theta_{\gamma_{\ell}, 2} \theta_{\gamma_{\mathrm{Q}}, 2} \mathcal{D}_{\gamma_{\mathrm{Q}^{-}}}(\bar{\phi})+\gamma_{\ell} \theta_{\gamma_{\mathrm{Q}}, 3} \mathcal{D}_{\gamma_{\ell^{+} \mathrm{Q}^{--}}}(\bar{\phi})\right\}
\end{gathered}
$$

$$
\begin{aligned}
& \left.\phi_{\mathrm{m}} \partial_{\ell}^{2} \mathcal{D}_{\gamma}(\bar{\phi})\right|_{\ell \neq m}=s^{-1} \gamma_{\mathrm{m}}(|\gamma|-1) \cdot\left\{\theta_{\gamma_{\ell}, 3} \mathcal{D}_{\gamma_{\mathrm{m}+\ell--}}(\bar{\phi})\right. \\
& \left.-2 \theta_{\gamma_{\ell}, 2} \theta_{\gamma_{\mathrm{Q}}, 2} \mathcal{D}_{\gamma_{\mathrm{m}+\ell-\mathrm{Q}^{-}}}(\bar{\phi})+\theta_{\gamma_{\mathrm{Q}}, 3} \mathcal{D}_{\gamma_{\mathrm{m}^{+} \mathrm{Q}^{--}}}(\bar{\phi})\right\} \\
& \phi_{\ell}^{2} \partial_{\ell}^{2} \mathcal{D}_{\gamma}(\bar{\phi})=\left(\gamma_{\ell}-2\right)\left(\gamma_{\ell}-1\right) \theta_{\gamma_{\ell}, 3} \mathcal{D}_{\gamma}(\bar{\phi})-2\left(\gamma_{\ell}-1\right) \gamma_{\ell} \theta_{\gamma_{\ell}, 2} \theta_{\gamma_{\mathrm{Q}}, 2} \mathcal{D}_{\gamma_{\ell+\mathrm{Q}^{-}}}(\bar{\phi}) \\
& \quad+\gamma_{\ell}\left(\gamma_{\ell}+1\right) \theta_{\gamma_{\mathrm{Q}}, 3} \mathcal{D}_{\gamma_{\ell++} \mathrm{Q}^{--}}(\bar{\phi}),
\end{aligned}
$$

$$
\begin{aligned}
\left.\phi_{\mathrm{m}}^{2} \partial_{\ell}^{2} \mathcal{D}_{\gamma}(\bar{\phi})\right|_{\ell \neq \mathrm{m}}=\gamma_{\mathrm{m}}\left(\gamma_{\mathrm{m}}+1\right)\left\{\theta_{\gamma_{\ell}, 3} \mathcal{D}_{\gamma_{\mathrm{m}++\ell--}}(\bar{\phi})\right. \\
\left.\quad-2 \theta_{\gamma_{\ell}, 2} \theta_{\gamma_{\mathrm{Q}}, 2} \mathcal{D}_{\gamma_{\mathrm{m}++\ell-\mathrm{Q}^{-}}}(\bar{\phi})+\theta_{\gamma_{\mathrm{Q}}, 3} \mathcal{D}_{\gamma_{\mathrm{m}^{++} \mathrm{Q}^{--}}}(\bar{\phi})\right\}
\end{aligned}
$$

$$
\begin{aligned}
\phi_{\ell} \phi_{\mathrm{m}} \partial_{\ell}^{2} & \left.\mathcal{D}_{\gamma}(\bar{\phi})\right|_{\ell \neq \mathrm{m}}=\gamma_{\mathrm{m}}\left\{\left(\gamma_{\ell}-2\right) \theta_{\gamma_{\ell}, 3} \mathcal{D}_{\gamma_{\mathrm{m}+\ell^{-}}}(\bar{\phi})\right. \\
& \left.-2\left(\gamma_{\ell}-1\right) \theta_{\gamma_{\ell}, 2} \theta_{\gamma_{\mathrm{Q}}, 2} \mathcal{D}_{\gamma_{\mathrm{m}^{+} \mathrm{Q}^{-}}}(\bar{\phi})+\gamma_{\ell} \theta_{\gamma_{\mathrm{Q}}, 3} \mathcal{D}_{\gamma_{\mathrm{m}+\ell+Q^{--}}}(\bar{\phi})\right\}
\end{aligned}
$$

$$
\begin{aligned}
& \left.\partial_{\ell} \partial_{\mathrm{m}} \mathcal{D}_{\gamma}(\bar{\phi})\right|_{\ell \neq m}=s^{-2}(|\gamma|-1)(|\gamma|-2) \\
& \cdot\left[\theta_{\gamma_{\ell}, 2} \theta_{\gamma_{\mathrm{m}}, 2} \mathcal{D}_{\gamma_{\left(\ell^{-}\right)\left(\mathrm{m}^{-}\right)}}(\bar{\phi})-\theta_{\gamma_{\ell}, 2} \theta_{\gamma_{\mathrm{Q}}, 2} \mathcal{D}_{\gamma_{\left(\ell^{-}\right)\left(Q^{-}\right)}}(\bar{\phi})\right. \\
& \left.-\theta_{\gamma_{m}, 2} \theta_{\gamma_{Q}, 2} \mathcal{D}_{\gamma_{\left(m^{-}\right)\left(Q^{-}\right)}}(\bar{\phi})+\theta_{\gamma_{Q}, 3} \mathcal{D}_{\gamma_{Q^{--}}}(\bar{\phi})\right] \text {, }
\end{aligned}
$$

$$
\begin{aligned}
& \left.\phi_{\ell} \partial_{\ell} \partial_{\mathrm{m}} \mathcal{D}_{\gamma}(\bar{\phi})\right|_{\ell \neq \mathrm{m}}=\mathrm{s}^{-1}(|\gamma|-1) \\
& \begin{array}{l}
\cdot\left\{\left(\gamma_{\ell}-1\right)\left[\theta_{\gamma_{\ell}, 2} \theta_{\gamma_{\mathrm{m}}, 2} \mathcal{D}_{\gamma_{\mathrm{m}}-}(\bar{\phi})-\theta_{\gamma_{\ell}, 2} \theta_{\gamma_{\mathrm{Q}}, 2} \mathcal{D}_{\gamma_{\mathrm{Q}^{-}}}(\bar{\phi})\right]\right. \\
\left.-\gamma_{\ell}\left[\theta_{\gamma_{\mathrm{m}}, 2} \theta_{\gamma_{\mathrm{Q}}, 2} \mathcal{D}_{\gamma_{\gamma^{+} \mathrm{m}^{-\mathrm{Q}^{-}}}}(\bar{\phi})-\theta_{\gamma_{\mathrm{Q}}, 3} \mathcal{D}_{\gamma_{\ell^{+} \mathrm{Q}^{--}}}(\bar{\phi})\right]\right\},
\end{array}
\end{aligned}
$$




$$
\begin{aligned}
\phi_{\ell} \phi_{\mathrm{m}} \partial_{\ell} \partial_{\mathrm{m}} & \left.\mathcal{D}_{\gamma}(\bar{\phi})\right|_{\ell \neq m}= \\
& \left(\gamma_{\ell}-1\right)\left(\gamma_{\mathrm{m}}-1\right) \theta_{\gamma_{\ell}, 2} \theta_{\gamma_{\mathrm{m}}, 2} \mathcal{D}_{\gamma^{\prime}}(\bar{\phi})-\gamma_{\mathrm{m}}\left(\gamma_{\ell}-1\right) \theta_{\gamma_{\ell}, 2} \theta_{\gamma_{\mathrm{Q}}, 2} \mathcal{D}_{\gamma_{\mathrm{m}}+\mathrm{Q}^{-}} \\
& -\gamma_{\ell}\left(\gamma_{\mathrm{m}}-1\right) \theta_{\gamma_{\mathrm{m}, 2}, 2} \theta_{\gamma_{\mathrm{Q}}, 2} \mathcal{D}_{\gamma_{\ell^{+} \mathrm{Q}^{-}}}(\bar{\phi})+\gamma_{\ell} \gamma_{\mathrm{m}} \theta_{\gamma_{\mathrm{Q}}, 3} \mathcal{D}_{\gamma_{\ell^{+} \mathrm{m}} \mathrm{Q}^{--}}(\bar{\phi}),
\end{aligned}
$$

where

$$
\theta_{a, b}= \begin{cases}1 & \text { if } \quad a \geqslant b \\ 0 & \text { otherwise. }\end{cases}
$$

Now, projecting - by way of example - eq. (3.19) onto $V_{\alpha}$ yields

$$
\left\langle\mathrm{V}_{\alpha}, \partial_{\ell} \mathcal{D}_{\gamma}\right\rangle_{\kappa}=\mathrm{s}^{-1}(|\gamma|-1)\left[\theta_{\gamma_{\ell}, 2} \chi_{\alpha \gamma_{\ell^{-}}}-\theta_{\gamma_{\mathrm{Q}}, 2} \chi_{\alpha \gamma_{Q^{-}}}\right] .
$$

Analogously it be can done for all eqs. (3.18)- $\sqrt{3.31}$; we see indeed that projecting the whole function $\mathcal{L}_{\mathrm{FP}} \cdot \mathcal{P}_{n}$ onto $V_{\alpha}$ is just a matter of tedious yet simple algebra. We conclude that $\psi_{\alpha \gamma}$ can be expanded as a self-contained sum of contributions, each being proportional to some matrix element of $\chi$. However, we observe that

$$
\left[\oplus_{1}^{m_{1}} \ldots \oplus_{\mathrm{Q}-1}^{\mathrm{m}_{\mathrm{Q}-1}}\right] \cdot\left[\ominus_{1}^{\ell_{1}} \ldots \ominus_{\mathrm{Q}-1}^{\ell_{\mathrm{Q}-1}}\right] \cdot \gamma \in \Omega_{\mathrm{n}+\sum_{k} \mathrm{~m}_{k}-\sum_{k} \ell_{k}} \quad \text { for } \gamma \in \Omega_{\mathrm{n}} \text { and }\left\{\ell_{k}<\gamma_{k}\right\}
$$

If $\max _{k} \operatorname{deg}\left\{A_{k}\right\}=m_{A}$, then the action of the drift term on $\mathcal{P}_{n}$ mixes Dirichlet distributions with index arrays in the bucket spaces $\Omega_{\mathfrak{n}-1}, \Omega_{\mathfrak{n}}, \ldots, \Omega_{\mathfrak{n}+m_{A}-1}$. Likewise, if $\max _{i, k} \operatorname{deg}\left\{B_{i k}\right\}=m_{B}$, then the action of the diffusion term on $\mathcal{P}_{n}$ mixes Dirichlet distributions with index arrays in the bucket spaces $\Omega_{n-2}, \Omega_{n-1}, \ldots, \Omega_{n+m_{B}-2}$. Accordingly, in order to compute the matrix $\psi$, we need to compute $\chi_{\alpha \gamma}$ for $|\alpha| \leqslant n$ and for $\gamma \in \Omega_{k}$ for some $k \in\left\{n-2, \ldots, n_{\max }\right\}$, with $n_{\max }=\max \left\{n+m_{A}-1, n+m_{B}-2\right\}$.

v) In order to work out eqs. (3.13)-(3.14, we first insert eq. $(1.9)$ into $J_{k}$ and extract the coefficient multiplying each $c_{\gamma}$, namely

$$
\mathrm{J}_{k}(\bar{\phi})=\sum_{\gamma \in \Omega_{n}} \mathrm{c}_{\gamma}\left\{A_{k}(\bar{\phi}) \mathcal{D}_{\gamma}(\bar{\phi})-\frac{1}{2} \sum_{i=1}^{\mathrm{Q}-1} \partial_{i}\left[\mathrm{~B}_{i k}(\bar{\phi}) \mathcal{D}_{\gamma}(\bar{\phi})\right]\right\} \equiv \sum_{\gamma \in \Omega_{n}} \Upsilon_{k \gamma}(\bar{\phi}) c_{\gamma} .
$$

We need to compute each matrix coefficient $\Upsilon_{k \gamma}(\bar{\phi})$ just for two sets of boundary points, namely $\Upsilon_{k \gamma}\left(\bar{\phi}_{k, m}\right)$ (in order to impose the boundary conditions on $\mathfrak{H}_{k}$ ) and $\Upsilon_{k \gamma}\left(\bar{\phi}_{\mathrm{Q}, \mathrm{m}}\right.$ ) (in order to impose the boundary conditions on $\left.\mathfrak{H}_{\mathrm{Q}}\right)$. The reader should notice that $\mathcal{D}_{\gamma}\left(\bar{\phi}_{\mathrm{km}}\right)=0$ unless $\gamma_{\mathrm{k}}=1$ as well as $\mathcal{D}_{\gamma}\left(\bar{\phi}_{\mathrm{Qm}}\right)=0$ unless $\gamma_{\mathrm{Q}}=1$. Since $\Upsilon_{\mathrm{k} \gamma}$ depends on both $\mathcal{D}_{\gamma}$ and $\left\{\partial_{i} \mathcal{D}_{\gamma}\right\}$, this means that $\Upsilon_{k \gamma}\left(\bar{\phi}_{\mathrm{km}}\right)=0$ unless $\gamma_{\mathrm{k}}=1,2$ and equally $\Upsilon_{\mathrm{k} \gamma}\left(\bar{\phi}_{\mathrm{Qm}}\right)=0$ unless $\gamma_{\mathrm{Q}}=1,2$. Therefore, we conclude that the only unknowns taking part in the boundary equations are those $c_{\gamma}$ which have at least one component $\gamma_{k}=1,2$ with $k=1, \ldots, Q$.

Remark 2. By now, it should be sufficiently clear what the pros and cons of projecting $\mathcal{P}$ onto a set of Dirichlet distributions are. We find it worthwhile summarizing them:

- the Dirichlet distributions $\left\{\mathcal{D}_{\gamma}\right\}_{\gamma \in \Omega_{\mathrm{n}}}$ are not orthogonal polynomials with respect to the scalar product $\langle\cdot, \cdot\rangle_{\kappa}$, yet they form a basis of $\overline{\mathcal{H}}_{n}$; 
- while the zeros of the orthogonal polynomials $\left\{\mathrm{V}_{\alpha}\right\}$ and $\left\{\mathrm{U}_{\alpha}\right\}$ are non-trivial algebraic varieties, the Dirichlet distributions $\left\{\mathcal{D}_{\gamma}\right\}_{\gamma \in \Omega_{n}}$ are non-negative on $\overline{\mathrm{T}}_{\mathrm{Q}}(\mathrm{s})$. This means that the positiveness of $\mathcal{P}_{n}$ relies entirely on the signs of the expansion coefficients $\left\{\mathbf{c}_{\gamma}\right\}$. If $\mathbf{c}_{\gamma} \geqslant 0 \forall \gamma \in \Omega_{n}$, then $\mathcal{P}_{n}$ can be statistically interpreted as a distributional mixture;

- if for too small values of $n$ the $R G$ approximation gives $\mathcal{P}_{n}(\bar{\phi})<0$ for $\bar{\phi}$ in some positive-measure subset of $\overline{\mathrm{T}}_{\mathrm{Q}}(\mathrm{s})$, it is anyway possible to obtain a decent (non-quasi-best) approximation of $\mathcal{P}$ by changing the sign of some coefficient $\mathrm{c}_{\gamma}$ and by subsequently renormalizing the whole vector $\mathrm{c}$;

- the differentiation rules of $\mathcal{D}_{\gamma}$ generate self-contained algebraic expressions involving Dirichlet distributions with different indices. Although $\psi$ is a dense matrix, it can be easily computed. Notice, however, that not only $\left|\Omega_{n}\right|$ inflates almost exponentially with $\mathrm{Q}$, but also the CPU time needed to compute $\chi_{\alpha \gamma}$ for a given pair $(\alpha, \gamma)$ blows up, since eq. 2.14 contains a non-factorizable multiple sum $\sum_{\beta \leqslant \alpha}=\sum_{\beta_{1}=0}^{\alpha_{1}} \cdots \sum_{\beta_{\mathrm{Q}-1}=0}^{\alpha_{\mathrm{Q}-1}}$

- the Dirichlet distribution $\mathcal{D}_{\gamma}$ vanishes on $\mathfrak{H}_{\mathrm{k}}$ unless $\gamma_{\mathrm{k}}=1$. It is therefore very simple to keep track of which terms are responsible for the behaviour of $\mathcal{P}_{\mathrm{n}}$ on $\partial \overline{\mathrm{T}}_{\mathrm{Q}}(\mathrm{s})$. Such a task would be a nightmare with any other polynomial basis.

\section{Example 1: binary voter model with zealots}

The binary voter model, introduced in [12, 13], can be considered as an archetype of agent-based models for opinion dynamics. Owing popularity to its exact solvability on a lattice in any dimension, the model has been studied in a number of variants. We refer the reader to [1] for a comprehensive review of the relevant literature. The microscopic dynamics of the model is simply defined. Agents carry a binary variable $v \in\{+1,-1\}$ and are selected at random for transitions. When an agent is selected, she flips her variable to that of a neighbour agent, also chosen at random. In a certain time the system collapses to a consensus state (all agents eventually share the same opinion), unless a stabilization mechanism is turned on. One possibility is to perturb the system by introducing zealots among the agents, i.e. special individuals who never change their opinion. Zealots in the context of the binary voter model have been originally proposed in [14]. If competing zealots with opposite opinions are present, consensus states are prevented as discussed in [7]. As far as we are concerned here, the binary voter model with zealots is of interest because

- it is a one-dimensional model, i.e. $\mathrm{Q}=2$;

- the FPE of the model can be solved exactly.

Both these features make it a simple case study to test the RG method. Let $\mathrm{N}$ denote the total number of agents, $Z_{ \pm}$the number of zealots with $v= \pm 1$ and $N_{ \pm}$the number of dynamic agents with $v= \pm 1$. Along with [7], we define $\phi=\mathrm{N}_{+} / \mathrm{N}, z_{ \pm}=\mathrm{Z}_{ \pm} / \mathrm{N}$ and $s=1-z_{+}-z_{-}$. Accordingly, it must be $0 \leqslant \phi \leqslant s$, i.e. $\overline{\mathrm{T}}_{\mathrm{Q}}(s)$ is just an interval in this case. The FPE of the model reads

$$
0=-\partial_{\phi}[A(\phi) P(\phi)]+\frac{1}{2} \partial_{\phi}^{2}[B(\phi) P(\phi)]=\partial_{\phi}\left\{-A(\phi) P(\phi)+\frac{1}{2} \partial_{\phi}[B(\phi) P(\phi)]\right\}=\partial_{\phi} J(\phi),
$$

with $\partial_{\phi}=\partial / \partial \phi$. The drift and diffusion coefficients are given by

$$
\begin{aligned}
& \mathrm{A}(\phi)=\left[z_{+} s-\phi(1-s)\right], \\
& \mathrm{B}(\phi)=\mathrm{N}^{-1}\left[\left(\phi+z_{+}\right)(s-\phi)+\phi\left(s+z_{-}-\phi\right)\right] .
\end{aligned}
$$


If one introduces the auxiliary variables $\delta=z_{+}-z_{-}, r=\sqrt{\delta^{2}+4 s}$ and $u_{ \pm}=s / 2-\delta / 4 \pm r / 4$, then the exact solution of the FPE [7] reads

$$
\mathcal{P}(\phi)=W \cdot\left[\left(\phi-\mathfrak{u}_{+}\right)\left(\phi-\mathfrak{u}_{-}\right)\right]^{\left(Z_{+}+Z_{-}-2\right) / 2}\left[1+\frac{r}{2 \phi-s-\frac{r-\delta}{2}}\right]^{(\delta / 2 r)\left(2 N-Z_{+}-Z_{-}\right)},
$$

with $W$ being a normalization constant such that $\int_{0}^{s} \mathrm{~d} \phi \mathcal{P}(\phi)=1$. When $z_{ \pm}=z$, the solution collapses to

$$
\mathcal{P}_{\text {sym }}(\phi)=W \cdot[z s+2 \phi(s-\phi)]^{\mathrm{Nz}-1} .
$$

Notice that $\mathcal{P}_{\text {sym }}$ is a polynomial with $\operatorname{deg}\left\{\mathcal{P}_{\text {sym }}\right\}=2 \mathrm{~N} z-2$, while $\mathcal{P}$ is a rational function for $z_{+} \neq z_{-}$. It should be observed that the condition $\hat{n}(\bar{\phi}) \cdot J(\bar{\phi})=0$ is meaningless for $Q=2$ since $\hat{n}$ is not defined at all. Indeed, $Q=2$ is a degenerate case: $J(\phi)=$ const. is a first integral of eq. (4.1) and eq. (1.3) is simply fulfilled provided we choose the constant to be zero. Now, a Dirichlet distribution with $Q=2$ is actually a beta distribution

$$
\mathcal{D}_{\left(\gamma_{1}, \gamma_{2}\right)}(\phi)=\frac{\Gamma\left(\gamma_{1}+\gamma_{2}\right)}{\Gamma\left(\gamma_{1}\right) \Gamma\left(\gamma_{2}\right)} s^{1-\gamma_{1}-\gamma_{2}} \phi^{\gamma_{1}-1}(s-\phi)^{\gamma_{2}-1}=B_{\gamma_{1} \gamma_{2}}(\phi),
$$

and the bucket space amounts in this case to

$$
\Omega_{n}=\{(n+1,1),(n, 2), \ldots,(2, n),(1, n+1)\}, \quad\left|\Omega_{n}\right|=\left(\begin{array}{c}
n+1 \\
n
\end{array}\right)=n+1 .
$$

By using the differentiation formulae reported in sect. 3 and some scratch paper, we can easily work out the matrix coefficients $\psi_{\alpha \gamma}=\left\langle\mathrm{V}_{\alpha},-\mathrm{A} \mathcal{D}_{\gamma}+2^{-1} \partial_{\phi}\left[\mathrm{BD} \mathcal{D}_{\gamma}\right]\right\rangle_{\kappa}$, namely

$$
\left\langle\mathrm{V}_{\alpha},-\mathrm{A} \mathcal{D}_{\gamma}\right\rangle_{\kappa}=-z_{+} s \chi_{\alpha\left(\gamma_{1}, \gamma_{2}\right)}+s(1-s) \frac{\gamma_{1}}{\gamma_{1}+\gamma_{2}} \chi_{\alpha\left(\gamma_{1}+1, \gamma_{2}\right)},
$$

and

$$
\begin{aligned}
& \left\langle\mathrm{V}_{\alpha}, \frac{1}{2} \partial_{\phi}\left[\mathrm{B} \mathcal{D}_{\gamma}\right]\right\rangle_{\kappa}=\frac{1}{\mathrm{~N}}\left\{-2 s \frac{\gamma_{1}}{\gamma_{1}+\gamma_{2}} \chi_{\alpha\left(\gamma_{1}+1, \gamma_{2}\right)}+\frac{1}{2}\left(2 s-z_{+}+z_{-}\right) \chi_{\alpha\left(\gamma_{1}, \gamma_{2}\right)}\right. \\
& -\frac{s}{\gamma_{1}+\gamma_{2}}\left[\gamma_{1}\left(\gamma_{1}-1\right) \theta_{\gamma_{1}, 2} \chi_{\alpha\left(\gamma_{1}, \gamma_{2}\right)}-\gamma_{1}\left(\gamma_{1}+1\right) \theta_{\gamma_{2}, 2} \chi_{\alpha\left(\gamma_{1}+1, \gamma_{2}-1\right)}\right] \\
& +\frac{1}{2}\left(2 s-z_{+}+z_{-}\right)\left[\left(\gamma_{1}-1\right) \theta_{\gamma_{1}, 2} \chi_{\alpha\left(\gamma_{1}, \gamma_{2}\right)}-\gamma_{1} \theta_{\gamma_{2}, 2} \chi_{\alpha\left(\gamma_{1}+1, \gamma_{2}-1\right)}\right]
\end{aligned}
$$

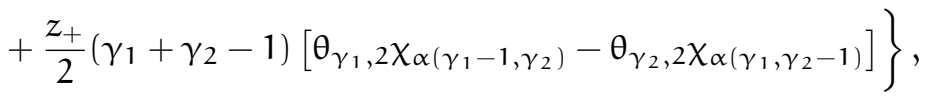

with $\gamma \in \Omega_{n}$ and $\alpha=0,1, \ldots, n$. Having coded $\psi$, we checked numerically that $v_{0}=1$ independently of $n$. The RG problem is therefore well posed: it is sufficient to impose the normalization condition eq. (1.10) to guarantee that $\Psi$ has maximal rank.

Numerical results are illustrated in Fig. 3 for both symmetric and asymmetric cases: the two plots on top show the exact solution $\mathcal{P}_{\text {sym }}$ and its $R G$ approximations for $N=1000, Z_{ \pm}=Z \in\{16,32\}$, $\kappa=(2,2)$ and a bunch of values of $n$; the central plots show results for the same physical setup, yet with asymmetric choices of the weight index array $k$; finally, those at the bottom show the exact solution $\mathcal{P}$ and its $R G$ approximations for $N=1000, Z_{+}=4, Z_{-} \in\{16,32\}$ and $k=(2,2)$. In Fig. 4. we report plots of the Dirichlet spectra obtained in the symmetric case with $K=(2,2)$. A few comments are in order: 

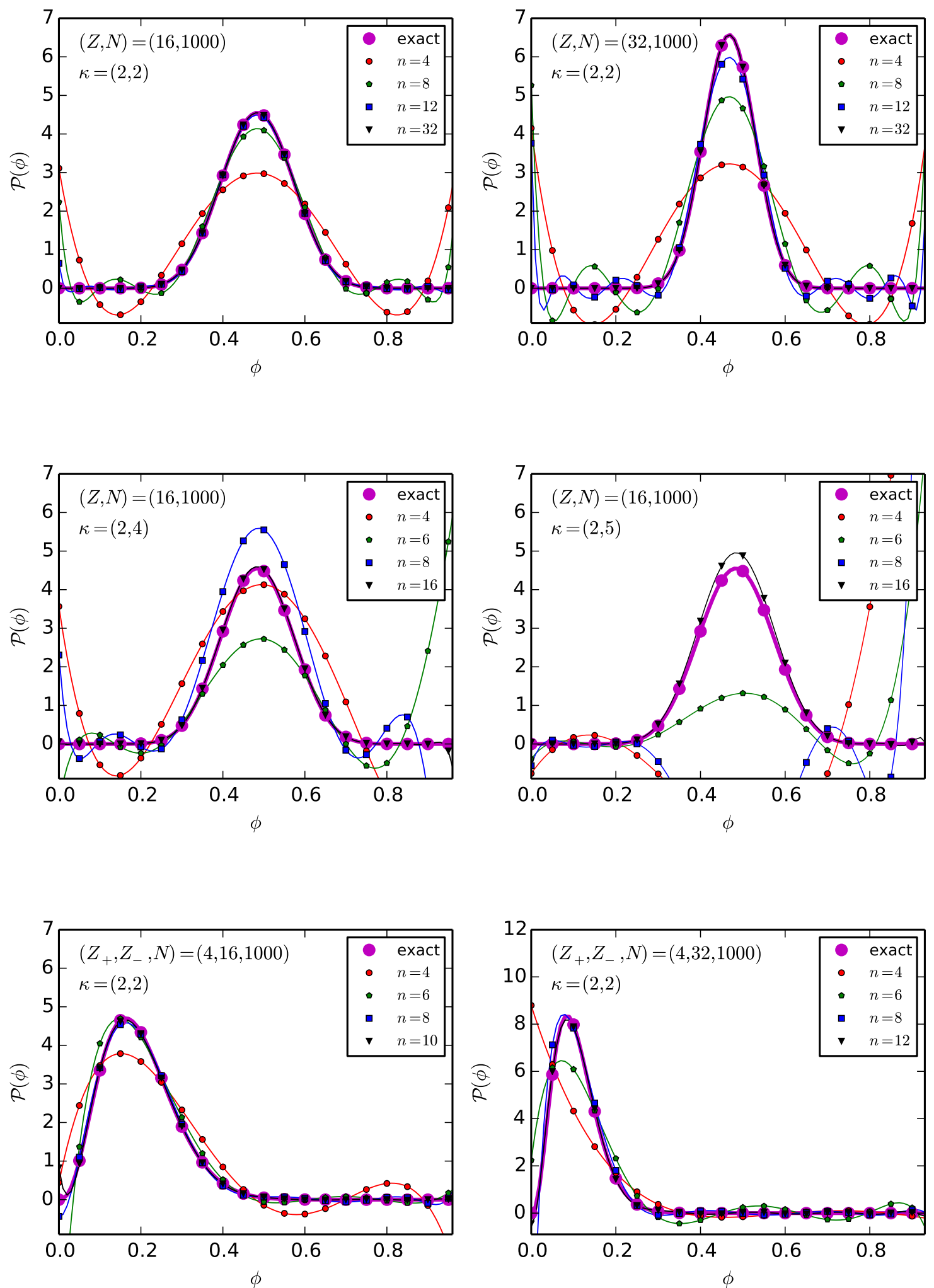

Figure 3 - RG approximations of the probability density of the binary voter model for various parameter sets. 

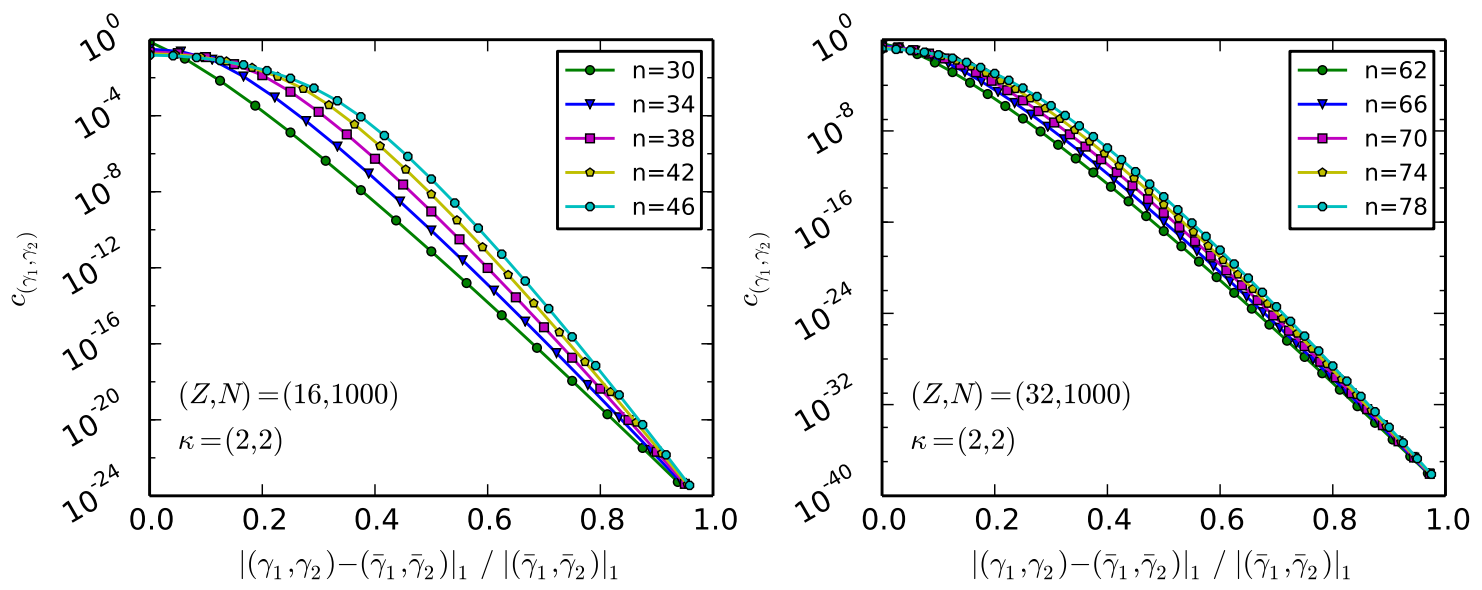

Figure 4 - Dirichlet spectra of the RG approximations.

- positiveness is violated at small values of $n$ for essentially all physical setups, but soon recovered at larger $n$;

- distributional convergence is reached at $n=2 \mathrm{Nz}-2$ in the symmetric case. It could not be otherwise: in order to exactly represent a polynomial $\mathrm{P}$ with $\operatorname{deg}\{\mathrm{P}\}=\mathrm{n}$ by another polynomial $\mathrm{Q}$, it must be $\operatorname{deg}\{\mathrm{Q}\} \geqslant \mathrm{n}$;

- convergence deteriorates in the symmetric case for $\kappa_{1} \neq \kappa_{2}$. Broadly speaking, the measure weight $\mathcal{D}_{K}$ overlaps with both $V_{\alpha}$ and $\mathcal{L}_{\mathrm{FP}} \cdot \mathcal{D}_{\gamma}$ in $\psi_{\alpha \gamma}$. If the mass of $\mathcal{P}$ concentrates in a given subset of $\bar{T}_{Q}(s)$, it is recommendable not to use a weight function whose mass concentrates elsewhere. Although this suggestion is only useful once $\mathcal{P}$ is known, symmetries should be taken into account in order to properly choose $\mathrm{k}$;

- the RG method works well also in the asymmetric case, where $\mathcal{P}$ is a rational function. Here, exact convergence is expected to be reached only asymptotically;

- the Dirichlet spectra look rather localized. Instead of ordering $\mathbf{c}_{\left(\gamma_{1}, \gamma_{2}\right)}$ according to the RLO, in Fig. 4 we plot data against the relative 1-norm distance of $\left(\gamma_{1}, \gamma_{2}\right)$ from

$$
\left(\bar{\gamma}_{1}, \bar{\gamma}_{2}\right)=\underset{\left(\gamma_{1}, \gamma_{2}\right) \in \Omega_{n}}{\operatorname{argmax}}\left\{\mathrm{c}_{\left(\gamma_{1}, \gamma_{2}\right)}\right\}=\left(\frac{\mathrm{n}}{2}+1, \frac{\mathrm{n}}{2}+1\right), \quad \mathrm{n} \text { even } .
$$

The outcome is evidently an exponential decrease for $\mathrm{n}=2 \mathrm{~N} z-2$, with an increasingly marked bending at larger values of $n$. An exponential behaviour is not surprising in consideration that $\mathcal{P}_{\text {sym }}$ is essentially a centered Gaussian distribution;

- the bending at $\mathrm{n}>2 \mathrm{Nz}-2$ is clearly due to the non-orthogonality of $\left\{\mathcal{D}_{\gamma}\right\}$.

\section{Example 2: multi-state voter model with zealots}

As a second case study for the RG method, we examine the multi-state voter model with zealots, a generalization of the binary version considered so far, where both dynamic agents and zealots carry an opinion $v \in\{1, \ldots, Q\}$. The ordering dynamics of the model with no zealots has been discussed in [15], while a variant with committed agents on a weighted network has been more recently studied 
in [16]. Here, we are interested in a simple formulation of the model with $\mathrm{N}$ agents on the complete graph, for which we expect the mean field description to work well. Let $N_{k}$ and $Z_{k}$ denote respectively the number of dynamic agents and zealots with $v=k$. We define $\phi_{k}=N_{k} / N, z_{k}=Z_{k} / N$ and $s=1-\sum_{\mathrm{k}=1}^{\mathrm{Q}-1} z_{\mathrm{k}}$. The FPE reads

$$
0=-\sum_{\ell=1}^{\mathrm{Q}-1} \partial_{\ell}\left[A_{\ell}(\phi) \mathrm{P}(\phi)\right]+\frac{1}{2} \sum_{\ell, \mathrm{m}=1}^{\mathrm{Q}-1} \partial_{\ell} \partial_{\mathrm{m}}\left[\mathrm{B}_{\ell \mathrm{m}}(\phi) \mathrm{P}(\phi)\right]
$$

with drift and diffusion coefficients given by

$$
\begin{aligned}
\mathrm{A}_{\ell}(\bar{\phi}) & =z_{\ell} s-(1-s) \phi_{\ell} \\
\mathrm{B}_{\ell \mathrm{m}}(\bar{\phi}) & =\frac{\delta_{\ell \mathfrak{m}}}{\mathrm{N}}\left[\left(\phi_{\ell}+z_{\ell}\right)\left(s-\phi_{\ell}\right)+\phi_{\ell}\left(1-z_{\ell}-\phi_{\ell}\right)\right]-\frac{1-\delta_{\ell \mathfrak{m}}}{\mathrm{N}}\left[2 \phi_{\ell} \phi_{\mathrm{m}}+z_{\ell} \phi_{\mathrm{m}}+z_{\mathrm{m}} \phi_{\ell}\right] .
\end{aligned}
$$

To the best of our knowledge, no analytic solution of the FPE is known in the literature. Therefore, the results of the RG method can be only compared to numerical simulations. Similar to the previous section, the derivation of $\psi_{\alpha \gamma}$ requires a modest algebraic effort. We have indeed

$$
\begin{aligned}
& \left\langle\mathrm{V}_{\alpha},-\sum_{\ell=1}^{\mathrm{Q}-1} \partial_{\ell}\left[A_{\ell} \mathcal{D}_{\gamma}\right]\right\rangle_{\kappa}=(\mathrm{Q}-1)(1-\mathrm{s})\left\langle\mathrm{V}_{\alpha}, \mathcal{D}_{\gamma}\right\rangle_{\kappa}+(1-\mathrm{s}) \sum_{\ell=1}^{\mathrm{Q}-1}\left\langle\mathrm{~V}_{\alpha}, \phi_{\ell} \partial_{\ell} \mathcal{D}_{\gamma}\right\rangle_{\kappa} \\
& -s \sum_{\ell=1}^{\mathrm{Q}-1} z_{\ell}\left\langle\mathrm{V}_{\alpha}, \partial_{\ell} \mathcal{D}_{\gamma}\right\rangle_{\kappa} \\
& \left\langle\mathrm{V}_{\alpha}, \frac{1}{2} \sum_{\mathrm{m}, \ell=1}^{\mathrm{Q}-1} \partial_{\ell} \partial_{\mathrm{m}}\left[\mathrm{B}_{\ell \mathrm{m}} \mathcal{D}_{\gamma}\right]\right\rangle_{\kappa}=-\frac{\mathrm{Q}(\mathrm{Q}-1)}{\mathrm{N}}\left\langle\mathrm{V}_{\alpha}, \mathcal{D}_{\gamma}\right\rangle_{\kappa}-\frac{2 \mathrm{Q}}{\mathrm{N}} \sum_{\ell=1}^{\mathrm{Q}-1}\left\langle\mathrm{~V}_{\alpha}, \phi_{\ell} \partial_{\ell} \mathcal{D}_{\gamma}\right\rangle_{\kappa} \\
& +\frac{1}{\mathrm{~N}} \sum_{\ell=1}^{\mathrm{Q}-1}\left(1+\mathrm{s}-\mathrm{Q} z_{\ell}\right)\left\langle\mathrm{V}_{\alpha}, \partial_{\ell} \mathcal{D}_{\gamma}\right\rangle_{\kappa}-\frac{1}{\mathrm{~N}} \sum_{\ell=1}^{\mathrm{Q}-1}\left\langle\mathrm{~V}_{\alpha}, \phi_{\ell}^{2} \partial_{\ell}^{2} \mathcal{D}_{\gamma}\right\rangle_{\kappa} \\
& +\frac{1}{2 \mathrm{~N}} \sum_{\ell=1}^{\mathrm{Q}-1}\left(1+\mathrm{s}-2 z_{\ell}\right)\left\langle\mathrm{V}_{\alpha}, \phi_{\ell} \partial_{\ell}^{2} \mathcal{D}_{\gamma}\right\rangle_{\kappa}+\frac{\mathrm{s}}{2 \mathrm{~N}} \sum_{\ell=1}^{\mathrm{Q}-1} z_{\ell}\left\langle\mathrm{V}_{\alpha}, \partial_{\ell}^{2} \mathcal{D}_{\gamma}\right\rangle_{\kappa} \\
& -\frac{1}{2 \mathrm{~N}} \sum_{\ell \neq m}^{1 \ldots Q}\left[2\left\langle\mathrm{~V}_{\alpha}, \phi_{\ell} \phi_{\mathrm{m}} \partial_{\ell} \partial_{\mathrm{m}} \mathcal{D}_{\gamma}\right\rangle_{\kappa}+z_{\ell}\left\langle\mathrm{V}_{\alpha}, \phi_{\mathrm{m}} \partial_{\ell} \partial_{\mathrm{m}} \mathcal{D}_{\gamma}\right\rangle_{\kappa}+z_{\mathrm{m}}\left\langle\mathrm{V}_{\alpha}, \phi_{\ell} \partial_{\ell} \partial_{\mathrm{m}} \mathcal{D}_{\gamma}\right\rangle_{\mathrm{k}}\right],
\end{aligned}
$$

and we simply need to express the various scalar products in terms of the matrix elements of $\chi$ via eqs. 3.18)-3.31. To give a feeling of the goodness of the approximation, in Fig. 5 we qualitatively compare the histogram of the probability density obtained from Monte Carlo simulations of the model (top left) and the RG approximation (top right) for a physical setup with $Q=3, N=1000, Z_{1}=Z_{2}=$ $Z_{3}=4$ and $R G$ parameters $n=12, n_{b c}=20$ and $k=(2,2,2)$.

It is interesting to examine how much $\mathcal{P}_{\mathfrak{n}}$ depends upon the number $n_{\mathrm{bc}}$ of boundary conditions. 2-norm distances can be easily evaluated once the RG coefficients are known. If $\mathcal{P}_{n}^{(1)}=\sum_{\gamma \in \Omega_{n}} c_{\gamma}^{(1)} \mathcal{D}_{\gamma}$ and $\mathcal{P}_{\mathrm{n}}^{(2)}=\sum_{\gamma \in \Omega_{\mathrm{n}}} \mathrm{c}_{\gamma}^{(2)} \mathcal{D}_{\gamma}$, then it can be shown that

$$
\left\|\mathcal{P}_{n}^{(1)}-\mathcal{P}_{n}^{(2)}\right\|_{2}^{2}=s^{1-Q} \sum_{\gamma, \eta \in \Omega_{n}}\left[c_{\gamma}^{(1)}-c_{\gamma}^{(2)}\right]\left[c_{\eta}^{(1)}-c_{\eta}^{(2)}\right] \frac{\Gamma(|\gamma|) \Gamma(|\eta|)}{\Gamma(|\gamma|+|\eta|-Q)} \prod_{k=1}^{Q} \frac{\Gamma\left(\gamma_{k}+\eta_{k}-1\right)}{\Gamma\left(\gamma_{k}\right) \Gamma\left(\eta_{k}\right)} .
$$



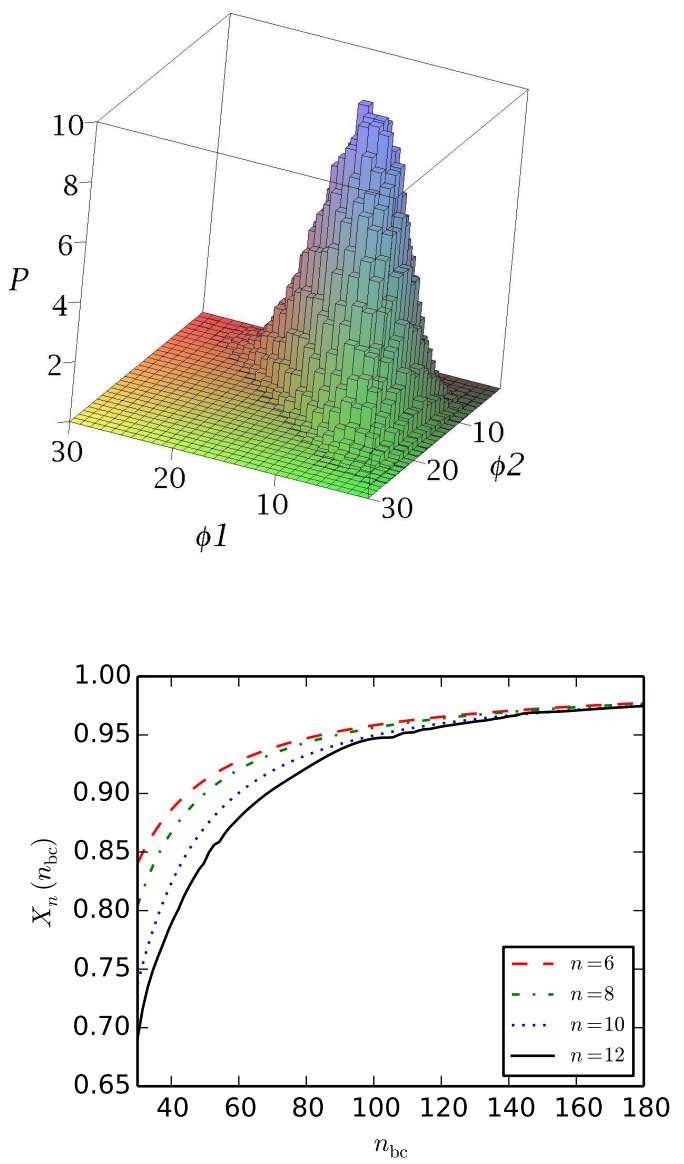

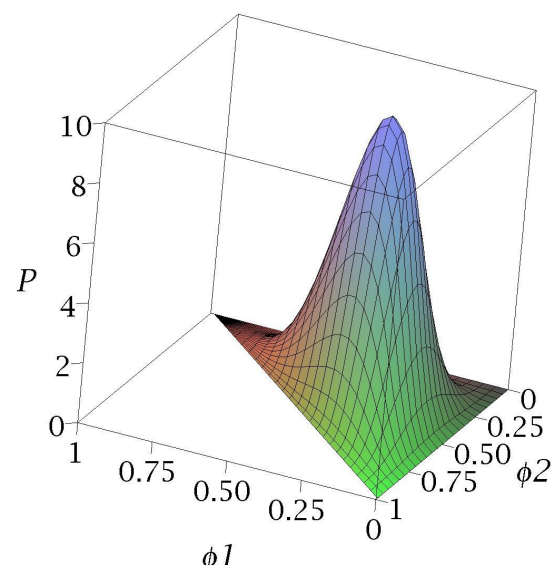

\begin{tabular}{r|c}
\hline \hline $\mathrm{n}$ & $\log _{10} \operatorname{cond}\left\{\Psi^{\mathrm{T}} \cdot \Psi\right\}$ \\
\hline 6 & $10.67(1)$ \\
8 & $14.85(1)$ \\
10 & $19.17(1)$ \\
12 & $23.69(1)$ \\
14 & $28.24(1)$ \\
16 & $32.73(1)$ \\
18 & $37.16(1)$ \\
\hline \hline
\end{tabular}

Figure 5 - (top left) Probability density of the multi-state voter model from Monte Carlo simulations (axes have been rescaled so as to host a 30-bins histogram with unitary bin size). (top right) RG probability density with parameters $n=12, n_{\mathrm{bc}}=20$ and $k=(2,2,2)$. (bottom left) Sensitivity of $\mathcal{P}_{n}$ to the number $n_{\mathrm{bc}}$ of boundary conditions, see eq. 5.7. (bottom right) condition number of $\Psi^{\mathrm{T}} \cdot \Psi$. All plots refer to a physical setup with $Q=3, N=1000, Z_{1}=Z_{2}=Z_{3}=4$.

In order to assess the sensitivity of $\mathcal{P}_{n}$ to $n_{\mathrm{bc}}$, we could look at $\left\|\left.\mathcal{P}_{n}\right|_{n_{\mathrm{bc}}+2}-\left.\mathcal{P}_{n}\right|_{n_{\mathrm{bc}}}\right\|_{2}^{2}$ as a function of $n_{\mathrm{bc}}$. Unfortunately, this quantity depends strongly on the polynomial degree $n$, hence it becomes difficult to compare distances corresponding to different values of $n$. A smoother behaviour is displayed by the distance ratio

$$
X_{n}\left(n_{b c}\right)=\sqrt{\frac{\left\|\left.\mathcal{P}_{n}\right|_{n_{\mathrm{bc}}+2}-\left.\mathcal{P}_{n}\right|_{n_{\mathrm{bc}}}\right\|_{2}^{2}}{\left\|\left.\mathcal{P}_{n}\right|_{n_{\mathrm{bc}}}-\left.\mathcal{P}_{n}\right|_{n_{\mathrm{bc}}-2}\right\|_{2}^{2}}} \quad\left(\text { notice that } \lim _{n_{\mathrm{bc}} \rightarrow \infty} X_{n}\left(n_{\mathrm{bc}}\right)=1\right),
$$

which we plot against $n_{b c}$ in Fig. 5 (bottom left), once more for $Q=3, N=1000, Z_{1}=Z_{2}=Z_{3}=4$ and $K=(2,2,2)$. For $n>12$, limits of our computer implementation emerge: $X_{n}\left(n_{b c}\right)$ becomes numerically unstable due to large cancellations occurring when subtracting the coefficients $c_{\gamma}$, hence we give up reporting it. Anyway, the plot shows that solutions of higher degree are more sensitive to the number of boundary conditions. This looks natural if one considers that the larger $n$ the more $\mathcal{P}_{n}$ fluctuates on the boundary hypersurfaces: in order to gentle the orthogonal probability flux crossing the boundary, this must be forced to vanish at more and more boundary points. By construction $X_{n}$ carries no information about the overall scale of the 2 -norm distances. This turns out to be very small for all $n$ 
and $n_{b c} \gtrsim 10$ (this estimate is likely to increase for larger values of $Q$ ).

It is likewise interesting to look at the condition number of $\Psi^{\mathrm{T}} . \Psi$. As the table in Fig. 5 (bottom right) shows, this blows up exponentially as $n$ increases, while it is rather insensitive to $n_{b c}$ (the uncertainty reported in the table measures the variation range for $20 \leqslant n_{\mathrm{bc}} \leqslant 200$ ). The exponential enhancement with $n$ requires a robust algorithm in order to perform the matrix inversion, as already observed in sect. 3.

Finally, the above discussion concerns only the analytic properties of $\mathcal{P}_{n}$. In order to make a quantitative comparison between $\mathcal{P}_{\mathrm{n}}$ and the empirical probability density obtained from the Monte Carlo (MC) simulation of the multi-state voter model, we can look at the respective distributional moments. Those of $\mathcal{P}_{\mathrm{n}}$ can be easily worked-out and exactly expressed as functions of the coefficients $\left\{c_{\gamma}\right\}$. In particular, the first two moments are given by

$$
\begin{aligned}
& \mathbb{E}\left[\phi_{k} \mid \mathcal{P}_{n}\right]=s \sum_{\gamma \in \Omega_{n}} c_{\gamma} \frac{\gamma_{k}}{|\gamma|}, \\
& \mathbb{E}\left[\phi_{k}^{2} \mid \mathcal{P}_{n}\right]=s^{2} \sum_{\gamma \in \Omega_{n}} c_{\gamma} \frac{\gamma_{k}\left(\gamma_{k}+1\right)}{|\gamma|(|\gamma|+1)}, \quad \mathbb{E}\left[\phi_{j} \phi_{k} \mid \mathcal{P}_{n}\right]_{j \neq k}=s^{2} \sum_{\gamma \in \Omega_{n}} c_{\gamma} \frac{\gamma_{j} \gamma_{k}}{|\gamma|(|\gamma|+1)} .
\end{aligned}
$$

Numerical estimates look rather stable against changes of $n$ and $n_{b c}$. For physical parameters as above all $\mathrm{RG}$ approximations give $\mathbb{E}\left[\phi_{\mathrm{k}}\right]_{\mathrm{RG}}=0.32933 \ldots=\mathrm{s} / 3$. However, the first moment is not indicative, as it just results from the symmetry of the setup. In order to make a real comparison, we have to look at the second moments. Our best estimates from RG approximations are $\mathbb{E}\left[\phi_{k}^{2}\right]_{R G}=0.1253 \ldots$ to be compared to $\mathbb{E}\left[\phi_{\mathrm{k}}^{2}\right]_{\mathrm{MC}}=0.124(1)$ and for $j \neq k, \mathbb{E}\left[\phi_{j} \phi_{k}\right]_{\mathrm{RG}}=0.1000 \ldots$ to be compared to $\mathbb{E}\left[\phi_{j} \phi_{k}\right]_{\mathrm{MC}}=0.1004(5)$. As can be seen, results are in very good agreement.

\section{Symmetry considerations}

The symmetry group of the simplex is the symmetric group. Since the implementation of the RG method becomes numerically demanding at large $Q$, it is worthwhile discussing if and how permutational symmetries can help reduce the computational work load.

\subsection{Permutational symmetry of the coefficients $\chi_{\alpha \gamma}$}

We first observe that the choice of the weight index array $\mathrm{k}$ is totally arbitrary, yet different values of it correspond to different orthogonal bases. A convenient option is the isotropic one, namely

$$
\mathrm{K}_{\mathrm{iso}}=(\underbrace{\hat{k}, \ldots, \hat{\mathrm{k}}}_{\mathrm{Q}-1 \text { times }}, \overline{\mathrm{K}}) \text {, }
$$

which for any $\mathrm{Q}$ depends only on two integer values $\hat{\kappa}$ and $\bar{\kappa}$. If we denote by $S_{\mathrm{Q}-1}$ the set of permutations of $\{1, \ldots, Q-1\}>$ and for $\sigma \in S_{Q-1}$ we define $\sigma \cdot \bar{\phi} \equiv\left(\phi_{\sigma(1)}, \ldots, \phi_{\sigma(Q-1)}\right), \sigma \cdot \alpha=$ $\left(\alpha_{\sigma(1)}, \ldots, \alpha_{\sigma(\mathrm{Q}-1)}\right)$ for $\alpha \in \mathbb{N}_{0}^{\mathrm{Q}-1}$ and $\sigma \cdot \gamma=\left(\gamma_{\sigma(1)}, \ldots, \ldots, \gamma_{\sigma(\mathrm{Q}-1)}, \gamma_{\mathrm{Q}}\right)$ for $\gamma \in \mathbb{N}^{\mathrm{Q}}$, then we immediately see that $\mathcal{D}_{\mathrm{K}_{\text {iso }}}(\sigma \cdot \bar{\phi})=\mathcal{D}_{\text {Kiso }}(\bar{\phi})$. For any other choice of the index array, it holds

$$
\begin{aligned}
\mathcal{D}_{\gamma}(\sigma \cdot \bar{\phi}) & =\frac{\Gamma(|\gamma|)}{\prod_{m=1}^{\mathrm{Q}} \Gamma\left(\gamma_{\mathrm{m}}\right)} s^{1-|\gamma|}\left[\prod_{m=1}^{\mathrm{Q}-1} \phi_{\sigma(\mathrm{m})}^{\gamma_{\mathrm{m}}}\right](s-|\bar{\phi}|)^{\gamma / \mathrm{Q}} \\
& =\frac{\Gamma(|\gamma|)}{\prod_{\mathrm{m}=1}^{\mathrm{Q}} \Gamma\left(\gamma_{\mathrm{m}}\right)} s^{1-|\gamma|}\left[\prod_{m=1}^{\mathrm{Q}-1} \phi_{\mathrm{m}}^{\gamma_{\sigma^{-1}(m)}}\right](s-|\bar{\phi}|)^{\gamma_{\mathrm{Q}}}=\mathcal{D}_{\sigma^{-1} \cdot \gamma}(\bar{\phi}),
\end{aligned}
$$


with $\sigma^{-1}$ denoting the inverse permutation of $\sigma$. Remarkably, a property analogous to eq. 6.2 is also fulfilled by the orthogonal polynomials provided $\mathrm{K}=\mathrm{K}_{\mathrm{iso}}$, namely

Proposition 3. If $\mathrm{k}=\kappa_{\text {iso, }}$ then $\mathrm{V}_{\alpha}(\sigma \cdot \bar{\phi})=\mathrm{V}_{\sigma^{-1} \cdot \alpha}(\bar{\phi}), \mathrm{U}_{\alpha}(\sigma \cdot \bar{\phi})=\mathrm{U}_{\sigma^{-1} \cdot \alpha}(\bar{\phi})$ and $\mathrm{f}_{\sigma^{\prime} \cdot \alpha}=\mathrm{f}_{\alpha}$ for any $\alpha \in \mathbb{N}_{0}^{\mathrm{Q}-1}, \sigma \in \mathrm{S}_{\mathrm{Q}-1}$ and $\bar{\phi} \in \overline{\mathrm{T}}_{\mathrm{Q}}(\mathrm{s})$.

Proof. With regard to $V_{\alpha}$, we first notice by direct inspection that

$$
v_{\alpha(\sigma \cdot \beta)}(\kappa)=v_{\left(\sigma^{-1} \cdot \alpha\right) \beta}\left(\sigma^{-1} \cdot \kappa\right) .
$$

Therefore, we have

$$
\begin{aligned}
V_{\alpha}(\sigma \cdot \bar{\phi}) & =\sum_{\beta \leqslant \alpha} v_{\alpha \beta}(\kappa)(\sigma \cdot \bar{\phi})^{\beta}=\sum_{\beta \leqslant \alpha} v_{\alpha \beta}(\kappa) \bar{\phi}^{\sigma^{-1} \cdot \beta}=\sum_{\sigma \cdot \beta \leqslant \alpha} v_{\alpha(\sigma \cdot \beta)}(\kappa) \bar{\phi}^{\beta} \\
& =\sum_{\sigma \cdot \beta \leqslant \alpha} v_{\sigma^{-1} \cdot \alpha \beta}\left(\sigma^{-1} \cdot \kappa\right) \bar{\phi}^{\beta} .
\end{aligned}
$$

Moreover,

$$
\sum_{\sigma \cdot \beta \leqslant \alpha}=\sum_{\beta_{\sigma(1)}=0}^{\alpha_{1}} \ldots \sum_{\beta_{\sigma(d)}=0}^{\alpha_{d}}=\sum_{\beta_{1}=0}^{\alpha_{\sigma-1}(1)} \cdots \sum_{\beta_{d}=0}^{\alpha_{\sigma-1}(\mathrm{~d})}=\sum_{\beta \leqslant \sigma^{-1} \cdot \alpha}
$$

whence we conclude

$$
V_{\alpha}(\sigma \cdot \bar{\phi})=\sum_{\beta \leqslant \sigma^{-1} \cdot \alpha} v_{\left(\sigma^{-1} \cdot \alpha\right) \beta}(\sigma \cdot \kappa) \bar{\phi}^{\beta}=V_{\sigma^{-1} \cdot \alpha}(\bar{\phi}) \text { if } \quad \sigma \cdot \kappa=\kappa .
$$

Analogously, we have

$$
\begin{aligned}
\mathrm{U}_{\alpha}(\sigma \cdot \bar{\phi}) & =\frac{1}{\mathcal{D}_{\kappa}(\sigma \cdot \bar{\phi})} \frac{\partial^{|\alpha|}}{\partial \phi_{\sigma(1)}^{\alpha_{1}} \ldots \partial \phi_{\sigma(\mathrm{d})}^{\alpha_{d}}}\left\{\prod_{m=1}^{\mathrm{Q}-1} \phi_{\sigma(\mathrm{m})}^{\alpha_{\mathrm{m}}+\kappa_{m}-1}(1-|\bar{\phi}|)^{|\alpha|+\kappa_{\mathrm{Q}}-1}\right\} \\
& =\frac{1}{\mathcal{D}_{\sigma^{-1} \cdot k}(\bar{\phi})} \partial_{\sigma^{-1} \cdot \alpha}^{|\alpha|}\left\{\prod_{m=1}^{\mathrm{Q}-1} \phi_{m}^{\alpha_{\sigma^{-1}(\mathrm{~m})}+\kappa_{\sigma^{-1}(\mathrm{~m})}-1}(1-|\bar{\phi}|)^{|\alpha|+\kappa_{Q}-1}\right\} \\
& =\mathrm{U}_{\sigma^{-1} \cdot \alpha}(\bar{\phi}) \text { if } \quad \sigma^{-1} \cdot \kappa=\kappa .
\end{aligned}
$$

It is trivially clear that $f_{\sigma \cdot \alpha}=f_{\alpha}$ if $k=\kappa_{\text {iso }}$.

From Prop. 3 it follows

$$
\begin{aligned}
& \chi_{\alpha \gamma}=\int_{\overline{\mathrm{T}}_{\mathrm{Q}}(\mathrm{s})} \mathrm{d} \bar{\phi} \mathrm{V}_{\alpha}(\bar{\phi}) \mathcal{D}_{\gamma}(\bar{\phi}) \mathcal{D}_{\mathrm{K}_{\mathrm{iso}}}(\bar{\phi}) \stackrel{\bar{\phi} \rightarrow \sigma \cdot \bar{\Phi}^{\prime}}{=} \int_{\overline{\mathrm{T}}_{\mathrm{Q}}(\mathrm{s})} \mathrm{d} \bar{\phi}^{\prime} \mathrm{V}_{\alpha}\left(\sigma \cdot \bar{\phi}^{\prime}\right) \mathcal{D}_{\gamma}\left(\sigma \cdot \bar{\phi}^{\prime}\right) \mathcal{D}_{\mathrm{K}_{\mathrm{iso}}}\left(\sigma \cdot \bar{\phi}^{\prime}\right) \\
& =\int_{\overline{\mathrm{T}}_{\mathrm{Q}}(s)} \mathrm{d} \bar{\phi}^{\prime} \mathrm{V}_{\sigma^{-1} \cdot \alpha}\left(\bar{\phi}^{\prime}\right) \mathcal{D}_{\sigma^{-1} \cdot \gamma}\left(\bar{\phi}^{\prime}\right) \mathcal{D}_{\kappa_{\text {iso }}}\left(\bar{\phi}^{\prime}\right)=\chi_{\left(\sigma^{-1} \cdot \alpha\right)\left(\sigma^{-1} \cdot \gamma\right)} \quad \text { if } \kappa=\kappa_{\text {iso }} .
\end{aligned}
$$

Accordingly, many matrix elements of $\chi$ are exactly the same, which explains the little-square structure of Fig. 1. In order to establish a convenient way of computing $x$, we introduce the partition set

$$
\mathfrak{P}_{\mathrm{n}, \mathrm{Q}-1}=\left\{\alpha \in \mathbb{N}_{\mathrm{O}}^{\mathrm{Q}-1}:|\alpha|=\mathrm{n} \text { and } \alpha_{1} \geqslant \alpha_{2} \geqslant \ldots \geqslant \alpha_{\mathrm{Q}-1}\right\},
$$

and for $\alpha \in \mathbb{N}_{0}^{\mathrm{Q}-1}$ the permutation set

$$
\Pi(\alpha)=\left\{\eta \in \mathbb{N}_{0}^{Q^{-1}}: \eta=\sigma \cdot \alpha \text { for some } \sigma \in S_{Q-1}\right\} .
$$




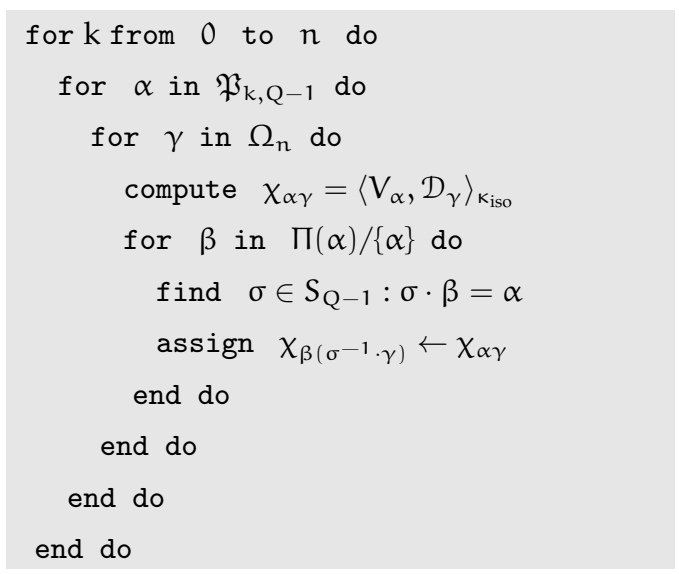

Figure 6 - A convenient recipe to compute $\chi$ when $\kappa=\kappa_{\text {iso }}$.

Obviously, if $\alpha$ has $m_{1}$ components equal to $a_{1}, \ldots, m_{r}$ components equal to $a_{r}$, such that

$$
m_{1}+\ldots+m_{r}=Q-1, \quad a_{1} m_{1}+\ldots+a_{r} m_{r}=|\alpha|,
$$

then it holds

$$
|\Pi(\alpha)|=\frac{(Q-1) !}{m_{1} ! \ldots m_{r} !}=\left(\begin{array}{c}
Q-1 \\
m_{1}, \ldots, m_{r}
\end{array}\right) .
$$

Partitions and permutations allow to decompose the index space of the orthogonal polynomials as a union of disjoint sets, namely

$$
\left\{\alpha \in \mathbb{N}_{0}^{\mathrm{Q}-1}:|\alpha| \leqslant n\right\}=\bigcup_{k=0}^{n} \bigcup_{\alpha \in \mathfrak{P}_{k, Q-1}} \Pi(\alpha) .
$$

In Fig. 6 we provide a recipe to compute $\chi$, which is based on the above set decomposition and works correctly since $\Omega_{\mathrm{n}}$ is permutationally closed. Moreover, it is well known since Euler's age [17] that $p_{n, Q-1} \equiv\left|\mathfrak{P}_{n, Q-1}\right|$ can be obtained from the generating function

$$
f(x)=\prod_{n=1}^{Q-1} \frac{1}{1-x^{k}}=\sum_{n=0}^{\infty} p_{n, Q-1} x^{k} .
$$

If we define the truncated Taylor expansion

$$
F_{n}(x)=\sum_{k=0}^{n} p_{k, Q-1} x^{k},
$$

then $\mathrm{q}_{\mathrm{n}, \mathrm{Q}-1}=\mathrm{F}_{\mathrm{n}}(1)$ represents the total number of matrix rows $\alpha$ for which $\chi_{\alpha \gamma}$ really needs to be computed. In Table 1, we report $q_{n, Q-1}$ for the first few values of $n$ and $Q$.

\subsection{Symmetric solutions of the Fokker-Planck equation}

If the FPE is symmetric under a subset of index permutations of $\bar{\phi}$, then its solution is expected to have the same symmetry (we assume here that no spontaneous symmetry breaking occurs). A permutationally symmetric FPE describes a system where no physical state is a priori favoured with 


\begin{tabular}{c|cccccccccccccc}
\hline \hline $\mathrm{n} \backslash \mathrm{Q}$ & 2 & 3 & 4 & 5 & 6 & 7 & 8 & 9 & 10 & 11 & 12 & 13 & 14 & 15 \\
\hline 0 & 1 & 1 & 1 & 1 & 1 & 1 & 1 & 1 & 1 & 1 & 1 & 1 & 1 & 1 \\
1 & 2 & 2 & 2 & 2 & 2 & 2 & 2 & 2 & 2 & 2 & 2 & 2 & 2 & 2 \\
2 & 4 & 4 & 4 & 4 & 4 & 4 & 4 & 4 & 4 & 4 & 4 & 4 & 4 & 4 \\
3 & 6 & 7 & 7 & 7 & 7 & 7 & 7 & 7 & 7 & 7 & 7 & 7 & 7 & 7 \\
4 & 9 & 11 & 12 & 12 & 12 & 12 & 12 & 12 & 12 & 12 & 12 & 12 & 12 & 12 \\
5 & 12 & 16 & 18 & 19 & 19 & 19 & 19 & 19 & 19 & 19 & 19 & 19 & 19 & 19 \\
6 & 16 & 23 & 27 & 29 & 30 & 30 & 30 & 30 & 30 & 30 & 30 & 30 & 30 & 30 \\
7 & 20 & 31 & 38 & 42 & 44 & 45 & 45 & 45 & 45 & 45 & 45 & 45 & 45 & 45 \\
8 & 25 & 41 & 53 & 60 & 64 & 66 & 67 & 67 & 67 & 67 & 67 & 67 & 67 & 67 \\
9 & 30 & 53 & 71 & 83 & 90 & 94 & 96 & 97 & 97 & 97 & 97 & 97 & 97 & 97 \\
10 & 36 & 67 & 94 & 113 & 125 & 132 & 136 & 138 & 139 & 139 & 139 & 139 & 139 & 139 \\
11 & 42 & 83 & 121 & 150 & 169 & 181 & 188 & 192 & 194 & 195 & 195 & 195 & 195 & 195 \\
12 & 49 & 102 & 155 & 197 & 227 & 246 & 258 & 265 & 269 & 271 & 272 & 272 & 272 & 272 \\
13 & 56 & 123 & 194 & 254 & 298 & 328 & 347 & 359 & 366 & 370 & 372 & 373 & 373 & 373 \\
14 & 64 & 147 & 241 & 324 & 388 & 433 & 463 & 482 & 494 & 501 & 505 & 507 & 508 & 508 \\
15 & 72 & 174 & 295 & 408 & 498 & 564 & 609 & 639 & 658 & 670 & 677 & 681 & 683 & 684 \\
\hline \hline
\end{tabular}

Table 1 - First coefficients $q_{n, Q-1}$.

respect to the others, a frequent case in phenomenological applications. For simplicity's sake, we assume that the FPE is maximally symmetric, i.e. it is symmetric under $\bar{\phi} \rightarrow \sigma \cdot \bar{\phi}, \forall \sigma \in S_{\mathrm{Q}-1}$. As an example, the reader could consider a multi-state voter model with zealots, where $Z_{k}=Z$ for $k=1, \ldots, Q$ and the network topology preserves the symmetry (for a non-trivial instance, see ref. [18]). Imposing that $\mathcal{P}_{n}$ is permutationally invariant yields

$$
\begin{aligned}
\mathcal{P}_{\mathrm{n}}(\sigma \cdot \bar{\phi}) & =\sum_{\gamma \in \Omega_{\mathrm{n}}} c_{\gamma} \mathcal{D}_{\gamma}(\sigma \cdot \bar{\phi})=\sum_{\gamma \in \Omega_{\mathrm{n}}} c_{\gamma} \mathcal{D}_{\sigma^{-1} \cdot \gamma}(\bar{\phi}) \\
& =\sum_{\gamma \in \Omega_{\mathrm{n}}} c_{\sigma \cdot \gamma} \mathcal{D}_{\gamma}(\bar{\phi})=\sum_{\gamma \in \Omega_{n}} c_{\gamma} \mathcal{D}_{\gamma}(\bar{\phi})=\mathcal{P}_{\mathfrak{n}}(\bar{\phi}),
\end{aligned}
$$

and since $\left\{\mathcal{D}_{\gamma}\right\}$ is a polynomial basis, we infer $\mathrm{c}_{\sigma \cdot \gamma}=\mathrm{c}_{\gamma}, \forall \gamma \in \Omega_{\mathrm{n}}$ and $\forall \sigma \in \mathrm{S}_{\mathrm{Q}-1}$. Of course, this result can be fruitfully used as a check of the numerical implementation of the RG method. Nevertheless, in this section we would like to discuss whether we can include such information even in the theoretical construction of the weak solution. In analogy with eqs. (6.9)- 6.10), we introduce the partition set

$$
\mathfrak{L}_{n}=\left\{\gamma \in \Omega_{n}: \gamma_{1} \geqslant \gamma_{2} \geqslant \ldots \geqslant \gamma_{Q-1}\right\},
$$

and for $\gamma \in \Omega_{n}$ the permutation set

$$
\Lambda(\gamma)=\left\{\eta \in \Omega_{n}: \eta=\sigma \cdot \gamma \text { for some } \sigma \in S_{Q-1}\right\} .
$$

If $\mathcal{P}_{\mathfrak{n}}$ is permutationally symmetric, then it can be written as

$$
\mathcal{P}_{\mathfrak{n}}(\bar{\phi})=\sum_{\gamma \in \mathfrak{L}_{\mathrm{n}}} \boldsymbol{c}_{\gamma} \sum_{\eta \in \Lambda(\gamma)} \mathcal{D}_{\mathfrak{\eta}}(\bar{\phi})=\sum_{\gamma \in \mathfrak{L}_{\mathrm{n}}} \hat{\boldsymbol{c}}_{\gamma} \frac{1}{|\Lambda(\gamma)|} \sum_{\eta \in \Lambda(\gamma)} \mathcal{D}_{\mathfrak{\eta}}(\bar{\phi})=\sum_{\gamma \in \mathfrak{L}_{\mathrm{n}}} \hat{c}_{\gamma} \mathcal{S} \cdot \mathcal{D}_{\gamma}(\bar{\phi}),
$$

where we have introduced the rescaled coefficient $\hat{c}_{\gamma}=|\Lambda(\gamma)| \mathbf{c}_{\gamma}$ and the symmetrized Dirichlet distribution $\mathcal{S} \cdot \mathcal{D}_{\gamma}=|\Lambda(\gamma)|^{-1} \sum_{\eta \in \Lambda(\gamma)} \mathcal{D}_{\eta}$. Eq. 6.19 tells us that a symmetric RG solution can be represented as a linear combination of symmetrized Dirichlet distributions (which are symmetric!). Can we 
reformulate the whole RG method so as to only make use of symmetrized Dirichlet distributions? The answer is affirmative, yet the reader should not undervalue technicalities.

i) As a preliminary observation, we argue that a symmetrized Dirichlet distribution faithfully decomposes into a basis of symmetrized orthogonal polynomials. To this aim, we first need to examine the permutational properties of the coefficients $\left\{\mathrm{d}_{\gamma \beta}\right\}$ connecting the Dirichlet basis $\left\{\mathcal{D}_{\gamma}\right\}$ to the Appel basis $\left\{\mathrm{U}_{\beta}\right\}$, see eq. 2.15. Under the isotropic assumption, a permutation of the components of $\bar{\phi}$ results in

$$
\mathcal{D}_{\gamma}(\sigma \cdot \bar{\phi})=\sum_{|\beta| \leqslant|\gamma|} \mathrm{d}_{\gamma \beta} \mathrm{u}_{\beta}(\sigma \cdot \bar{\phi})=\sum_{|\beta| \leqslant|\gamma|} \mathrm{d}_{\gamma \beta} \mathrm{u}_{\sigma^{-1} \cdot \beta}(\bar{\phi})=\sum_{|\beta| \leqslant|\gamma|} \mathrm{d}_{\gamma(\sigma \cdot \beta)} \mathrm{u}_{\beta}(\bar{\phi}) .
$$

However,

$$
\mathcal{D}_{\gamma}(\sigma \cdot \bar{\phi})=\mathcal{D}_{\sigma^{-1} \cdot \gamma}(\bar{\phi})=\sum_{|\beta| \leqslant|\gamma|} \mathrm{d}_{\left(\sigma^{-1} \cdot \gamma\right) \beta} \mathrm{u}_{\beta}(\bar{\phi}),
$$

whence we infer

$$
\sum_{|\beta| \leqslant|\gamma|} \mathrm{d}_{\left(\sigma^{-1} \cdot \gamma\right) \beta} \mathrm{u}_{\beta}(\bar{\phi})=\sum_{|\beta| \leqslant|\gamma|} \mathrm{d}_{\gamma(\sigma \cdot \beta)} \mathrm{U}_{\beta}(\bar{\phi}) .
$$

Since $\left\{U_{\beta}\right\}$ is a polynomial basis, we conclude that $d_{\gamma(\sigma \cdot \beta)}=d_{\left(\sigma^{-1} \cdot \gamma\right) \beta}$ (of course, this could have been equivalently obtained via the identity $\chi_{\alpha \gamma}=\chi_{\left(\sigma^{-1} \cdot \alpha\right)\left(\sigma^{-1} \cdot \gamma\right)}$, discussed in sect. 6.1). Then, we apply the symmetrization operator $\mathcal{S}$ to both sides of eq. (2.15), namely

$$
\begin{aligned}
\mathcal{S} \cdot \mathcal{D}_{\gamma}(\bar{\phi}) & =\frac{1}{|\Lambda(\gamma)|} \sum_{\eta \in \Lambda(\gamma)} \sum_{|\beta| \leqslant|\gamma|} \mathrm{d}_{\eta \beta} \mathrm{u}_{\beta}(\bar{\phi}) \\
& =\sum_{|\beta| \leqslant|\gamma|}\left[\frac{1}{|\Lambda(\gamma)|} \sum_{\eta \in \Lambda(\gamma)} \mathrm{d}_{\eta \beta}\right] \mathrm{u}_{\beta}(\bar{\phi})=\sum_{|\beta| \leqslant|\gamma|} \mathrm{e}_{\gamma \beta} \mathrm{u}_{\beta}(\bar{\phi}),
\end{aligned}
$$

with $e_{\gamma \beta} \equiv|\Lambda(\gamma)|^{-1} \sum_{\eta \in \Lambda(\gamma)} d_{\eta \beta}=\mathcal{S} \cdot d_{\gamma \beta}$. We can easily work out the permutational properties of the coefficients $\left\{e_{\gamma \beta}\right\}$. We have indeed

$$
e_{\gamma(\sigma \cdot \beta)}=\frac{1}{|\Lambda(\gamma)|} \sum_{\eta \in \Lambda(\gamma)} d_{\eta(\sigma \cdot \beta)}=\frac{1}{|\Lambda(\gamma)|} \sum_{\eta \in \Lambda(\gamma)} d_{\left(\sigma^{-1} \cdot \eta\right) \beta}=e_{\gamma \beta}
$$

as $\Lambda(\gamma)=\Lambda(\sigma \cdot \gamma)$ for any $\sigma \in S_{\mathrm{Q}-1}$. For the same reason, it holds

$$
e_{(\sigma \cdot \gamma) \beta}=\frac{1}{|\Lambda(\sigma \cdot \gamma)|} \sum_{\eta \in \Lambda(\sigma \cdot \gamma)} d_{\eta \beta}=\frac{1}{|\Lambda(\gamma)|} \sum_{\eta \in \Lambda(\gamma)} d_{\eta \beta}=e_{\gamma \beta} .
$$

Therefore, we conclude that $e_{\gamma(\sigma \cdot \beta)}=e_{(\sigma \cdot \gamma) \beta}=e_{\gamma \beta}$. By decomposing the set $\{|\beta| \leqslant|\gamma|\}$ according to eq. 6.13, we finally obtain

$$
\begin{aligned}
\mathcal{S} \cdot \mathcal{D}_{\gamma}(\bar{\phi}) & =\sum_{k=0}^{|\gamma|} \sum_{\beta \in \mathfrak{P}_{k, Q}-1} \sum_{\alpha \in \Pi(\beta)} e_{\gamma \alpha} \mathrm{u}_{\alpha}(\bar{\phi})=\sum_{k=0}^{|\gamma|} \sum_{\beta \in \mathfrak{P}_{k, Q}-1} e_{\gamma \beta} \sum_{\alpha \in \Pi(\beta)} \mathrm{u}_{\alpha}(\bar{\phi}) \\
& =\sum_{k=0}^{|\gamma|} \sum_{\beta \in \mathfrak{P}_{k, Q}-1} \hat{e}_{\gamma \beta} \mathcal{S} \cdot \mathrm{u}_{\beta}(\bar{\phi}),
\end{aligned}
$$


with $\hat{e}_{\gamma \beta} \equiv|\Pi(\beta)| e_{\gamma \beta}$. Eq. (6.26) tells us that since $\mathcal{S} \cdot \mathcal{D}_{\gamma}$ is a symmetric function, it decomposes faithfully into a set of symmetric polynomials $\left\{\mathcal{S} \cdot \mathrm{U}_{\beta}\right\}$. In addition, we notice that if $\alpha, \beta \in \mathbb{N}_{0}^{\mathrm{Q}-1}$, then either $\Pi(\alpha) \cap \Pi(\beta)=\emptyset$ or $\Pi(\alpha)=\Pi(\beta)$. Therefore, it makes sense to define

$$
\delta_{\Pi(\beta) \Pi(\gamma)}= \begin{cases}1 & \text { if } \Pi(\beta)=\Pi(\gamma), \\ 0 & \text { if } \Pi(\beta) \cap \Pi(\gamma)=\emptyset .\end{cases}
$$

Finally, we observe that

$$
\begin{aligned}
\left\langle\mathcal{S} \cdot \mathrm{v}_{\alpha}, \mathcal{S} \cdot \mathrm{u}_{\beta}\right\rangle_{\kappa} & =\frac{1}{\mid \Pi(\alpha)} \frac{1}{|\Pi(\beta)|} \sum_{\epsilon \in \Pi(\alpha)} \sum_{\eta \in \Pi(\beta)}\left\langle\mathrm{v}_{\epsilon}, \mathrm{u}_{\eta}\right\rangle_{\kappa} \\
& =\frac{1}{\mid \Pi(\alpha)} \frac{1}{|\Pi(\beta)|} \sum_{\epsilon \in \Pi(\alpha)} \sum_{\eta \in \Pi(\beta)} f_{\epsilon} \delta_{\epsilon \eta}=\frac{f_{\alpha}}{|\Pi(\alpha)|} \delta_{\Pi(\alpha) \Pi(\beta),}
\end{aligned}
$$

since $f_{\alpha}$ is permutationally invariant under the isotropic assumption. Now, projecting $\mathcal{S} \cdot \mathcal{D}_{\gamma}$ onto $\mathcal{S} \cdot V_{\alpha}$ with $|\alpha| \leqslant|\gamma|$ yields

$$
\begin{aligned}
\left\langle\mathcal{S} \cdot \mathrm{V}_{\alpha}, \mathcal{S} \cdot \mathcal{D}_{\gamma}\right\rangle_{\kappa} & =\sum_{k=0}^{|\gamma|} \sum_{\beta \in \mathfrak{P}_{k, Q}-1} \hat{e}_{\gamma \beta}\left\langle\mathcal{S} \mathrm{V}_{\alpha}, \mathcal{S} \mathrm{U}_{\beta}\right\rangle_{\kappa} \\
& =\sum_{k=0}^{|\gamma|} \sum_{\beta \in \mathfrak{P}_{k, Q}-1} \frac{\hat{e}_{\gamma \beta} \mathrm{f}_{\alpha}}{|\Pi(\alpha)|} \delta_{\Pi(\alpha) \Pi(\beta)}=e_{\gamma \alpha} \mathrm{f}_{\alpha},
\end{aligned}
$$

while the above scalar product vanishes for $|\alpha|>|\gamma|$. We conclude

$$
\left\langle\mathcal{S} \cdot V_{\alpha}, \mathcal{S} \cdot \mathcal{D}_{\gamma}\right\rangle_{\kappa}= \begin{cases}\chi_{\alpha \gamma} \cdot \overleftarrow{\mathcal{S}} & \text { if }|\alpha| \leqslant|\gamma| \\ 0 & \text { otherwise }\end{cases}
$$

where $\overleftarrow{\mathcal{S}}$ symmetrizes from the right over the index array $\gamma$

ii) We have already observed that the action of $\mathcal{L}_{\mathrm{FP}}$ mixes Dirichlet distributions with index arrays in different bucket spaces, corresponding to different polynomial degrees. Since the symmetrization operator $\mathcal{S}$ averages over permutations, we need to clarify how it behaves with respect to a shift of degree. This is needed in order to generate a dictionary of reference formulae analogous to eqs. 3.18)-(3.31). From a theoretical point of view, the problem originates from the fact that the index raising/lowering operators do not commute with permutations. Indeed, they fulfill the relations

$$
\begin{aligned}
& \oplus_{\ell} \cdot \sigma=\sigma \cdot \oplus_{\sigma(\ell)} \\
& \ominus_{\ell} \cdot \sigma=\sigma \cdot \ominus_{\sigma(\ell)}, \quad \ell=1, \ldots, Q-1, \quad \sigma \in S_{\mathrm{Q}-1} .
\end{aligned}
$$

As an example, we notice that the action of $\phi_{k}^{m} \partial_{k}^{n}$ on $\mathcal{S} \cdot \mathcal{D}_{\gamma}$ breaks the permutational symmetry of the symmetrized Dirichlet distribution and shifts its degree for $m \neq n$. If we then sum over $k$, the symmetry is recovered, but in general the result cannot be written anymore as a permutational average over $\Lambda(\gamma)$. With the same spirit by which we wrote down eqs. 3.18)-(3.31, we consider some specific cases. Two very simple ones are

$$
\begin{aligned}
& \sum_{\ell=1}^{\mathrm{Q}-1} \phi_{\ell} \mathcal{S} \cdot \mathcal{D}_{\gamma}(\bar{\phi})=\frac{1}{|\Lambda(\gamma)|} \sum_{\eta \in \Lambda(\gamma)}|\bar{\phi}| \mathcal{D}_{\eta}(\bar{\phi})=\frac{1}{|\Lambda(\gamma)|} \sum_{\eta \in \Lambda(\gamma)}(s+|\bar{\phi}|-s) \mathcal{D}_{\eta}(\bar{\phi}) \\
& =s \mathcal{S} \cdot \mathcal{D}_{\gamma}(\bar{\phi})-s \frac{\gamma_{\mathrm{Q}}}{|\gamma|} \mathcal{S} \cdot \mathcal{D}_{\gamma_{\mathrm{Q}^{+}}}(\bar{\phi}),
\end{aligned}
$$


and

$$
\begin{aligned}
& \sum_{\ell=1}^{\mathrm{Q}-1} \phi_{\ell} \partial_{\ell} \mathcal{S} \cdot \mathcal{D}_{\gamma}(\bar{\phi})=\frac{1}{|\Lambda(\gamma)|} \sum_{\eta \in \Lambda(\gamma)} \sum_{\ell=1}^{\mathrm{Q}-1}\left[\theta_{\eta_{\ell}, 2}\left(\eta_{\ell}-1\right) \mathcal{D}_{\eta}(\bar{\phi})-\theta_{\eta_{\mathrm{Q}}, 2} \eta_{\ell} \mathcal{D}_{\eta_{\ell}+_{\mathrm{Q}^{-}}}(\bar{\phi})\right] \\
& =\left[\sum_{\ell=1}^{\mathrm{Q}-1} \theta_{\gamma_{\ell}, 2}\left(\gamma_{\ell}-1\right)+\theta_{\gamma_{\mathrm{Q}}, 2}\left(\gamma_{\mathrm{Q}}-1\right)\right] \mathcal{S} \cdot \mathcal{D}_{\gamma}(\bar{\phi})-\theta_{\gamma_{\mathrm{Q}}, 2}(|\gamma|-1) \mathcal{S} \cdot \mathcal{D}_{\gamma_{\mathrm{Q}^{-}}}(\bar{\phi}) .
\end{aligned}
$$

As can be seen, in both cases the result is still a permutational average over $\Lambda(\gamma)$. However, let us consider the action of $\sum_{\mathrm{k}=1}^{\mathrm{Q}-1} \partial_{\mathrm{k}}$ on $\mathcal{S} \cdot \mathcal{D}_{\gamma}$. From eq. 3.19, we have

$$
\begin{aligned}
& \sum_{\ell=1}^{\mathrm{Q}-1} \partial_{\ell} \mathcal{S} \cdot \mathcal{D}_{\gamma}(\bar{\phi})=\frac{\mathrm{s}^{-1}(|\gamma|-1)}{|\Lambda(\gamma)|} \sum_{\eta \in \Lambda(\gamma)} \sum_{\ell=1}^{\mathrm{Q}-1}\left[\theta_{\eta_{\ell}, 2} \mathcal{D}_{\eta_{\ell-}}(\bar{\phi})-\theta_{\eta_{\mathrm{Q}}, 2} \mathcal{D}_{\eta_{\mathrm{Q}^{-}}}(\bar{\phi})\right] \\
& =\mathrm{s}^{-1}(|\gamma|-1)\left\{\left[\frac{1}{|\Lambda(\gamma)|} \sum_{\eta \in \Lambda(\gamma)} \sum_{\ell=1}^{\mathrm{Q}-1} \theta_{\eta_{\ell, 2}} \mathcal{D}_{\eta_{\eta^{-}}}(\bar{\phi})\right]-\theta_{\gamma_{\mathrm{Q}}, 2}(\mathrm{Q}-1) \mathcal{S} \cdot \mathcal{D}_{\gamma_{\mathrm{Q}^{-}}}(\bar{\phi})\right\} .
\end{aligned}
$$

In order to check that the sum in square brackets is permutationally invariant, it is sufficient to make use of eq. 6.32 and observe that $\theta_{\eta_{\ell}, 2} \mathcal{D}_{\eta_{\ell^{-}}}(\sigma \cdot \bar{\phi})=\theta_{\gamma_{\sigma(\ell), 2}} \mathcal{D}_{\sigma \cdot \gamma_{\sigma(\ell)-}}(\sigma \cdot \bar{\phi})=\theta_{\gamma_{\sigma(\ell), 2}} \mathcal{D}_{\gamma_{\sigma(\ell)-}}(\bar{\phi})$, which upon summing over $\ell$ becomes manifestly invariant. Since $\left|\eta_{\ell_{-}}\right|=n-1$ for $\eta \in \Lambda(\gamma)$, we can write

$$
\frac{1}{|\Lambda(\gamma)|} \sum_{\eta \in \Lambda(\gamma)} \sum_{\ell=1}^{\mathrm{Q}-1} \theta_{\eta_{\ell}, 2} \mathcal{D}_{\eta_{\ell-}}(\bar{\phi})=\sum_{\eta \in \mathcal{L}_{n-1}} \hat{g}_{\eta} \mathcal{S} \cdot \mathcal{D}_{\eta}(\bar{\phi}),
$$

since we have already shown in eq. 6.19 - by an argument that could be here repeated - that a polynomial, which we know to be symmetric, can be expanded as a linear combination of symmetrized Dirichlet distributions (in this case the index arrays live on $\mathcal{L}_{n-1}$ due to the degree shift produced by the differentiation). Determining the coefficients $\left\{\hat{g}_{\mathfrak{n}}\right\}$ analytically is non-trival and beyond the aims of this paper. Nevertheless, by projecting eq. (6.36) onto the symmetrized orthogonal polynomials and by making use of eq. (6.30), we obtain

$$
\sum_{\eta \in \mathcal{L}_{n-1}}\left(e_{\eta \alpha} f_{\alpha}\right) \hat{g}_{\eta}=\frac{1}{|\Lambda(\gamma)|} \sum_{\eta \in \Lambda(\gamma)} \sum_{\ell=1}^{\mathrm{Q}-1} \theta_{\eta_{\ell}, 2}\left\langle\mathcal{S} \cdot V_{\alpha}, \mathcal{D}_{\eta_{\ell-}}(\bar{\phi})\right\rangle_{\kappa}
$$

which can be numerically inverted.

Along the same line, all the symmetric counterparts of eqs. (3.18) - 3.31) can be worked out. Analytic expressions such as eqs. 6.33)-(6.34) effectively help save CPU time, while formulae requiring numerical inversions such as eq. 6.37) are of no benefit. Such cases require more sophisticated analyses, which we do not attempt here.

\section{Conclusions}

In this paper we have explored the possibility of representing the solution of the Fokker-Planck equation for many-variable steady-state birth-death systems as a linear combination of Dirichlet distributions. This idea was first suggested in [18], where a variant of the multi-state voter model with zealots over a community-based network [19] was studied. We have shown here that quasi-optimal coefficients for such a linear expansion can be generally obtained from a variant of the Ritz-Galerkin method for 
partial differential equations. As a test, we have applied the Dirichlet expansion successfully to the binary and multi-state voter models with zealots on a complete graph. Although Ritz-Galerkin techniques are widely employed in engineering applications, no adaptation to systems defined on the simplex has been ever considered in the literature, to the best of our knowledge.

We expect the domain of applicability of the method to go beyond that of voter models and to extend to a positive-measure subset of statistical physics. Applications could include variants of SIS model for epidemic spreading, naming games (a variant with committed agents has been recently studied in [20]) and other complex systems, only subject to the conditions that i) a steady-state distribution with positive variances exists and $i$ ) the drift and diffusion coefficients of the Fokker-Planck equation are polynomials. With regard to condition $i$ ), our proposal could be generalized by considering an expansion in Dirichlet distributions with linear coefficients depending on time, so as to allow for a treatment of the time-dependent Fokker-Planck equation. This would permit to describe the system while it relaxes to equilibrium. Nevertheless, systems with consensus-like exit states, which have attracted much attention in recent years, are anyway ruled out as finite-degree polynomials can never approximate a Dirac delta distribution. Concerning condition $i$ ), cases where the drift and diffusion coefficients are non-polynomial analytic functions could be maybe faced by expanding them in power series to some finite degree $m$ and by subsequently applying the Ritz-Galerkin method; this would generate a sequence $\left(\mathcal{P}_{n, m}\right)$ of solutions, whose convergence for $n, m \rightarrow \infty$ should be studied.

We conclude by recalling that in this work we have provided no theoretical arguments to show that the coerciveness condition of the Lax-Milgram theorem is generally fulfilled by a Fokker-Planck operator with polynomial diffusion matrix on the simplex. Without a general proof, the applicability of the method has to be checked on a case-by-case basis.

\section{Acknowledgments}

The computing resources used for our numerical study and the related technical support have been partly provided by the CRESCO/ENEAGRID High Performance Computing infrastructure and its staff [21]. CRESCO (Computational RESearch centre on COmplex systems) is funded by ENEA and by Italian and European research programmes.

\section{Appendix A Dirichlet integrals}

\section{A.1 Normalization of $\mathcal{D}_{\alpha}$}

It is worthwhile describing a simple technique to calculate polynomial integrals on the simplex by means of a specific example, namely the normalization coefficient of the Dirichlet distribution $\mathcal{D}_{\alpha}(\bar{\phi})=$ $\mathrm{Z}_{\mathcal{D}}(\alpha, s)^{-1} \phi_{1}^{\alpha_{1}} \ldots \phi_{\mathrm{Q}-1}^{\alpha_{\mathrm{Q}-1}}(s-|\bar{\phi}|)^{\alpha_{\mathrm{Q}}}$. Specifically, the integral that we aim at calculating is

$$
\mathrm{Z}_{\mathcal{D}}(\alpha, s)=\int_{\overline{\mathrm{T}}_{\mathrm{Q}}(s)} \mathrm{d} \bar{\phi} \phi_{1}^{\alpha_{1}} \ldots \phi_{\mathrm{Q}-1}^{\alpha_{\mathrm{Q}-1}}(s-|\bar{\phi}|)^{\alpha_{\mathrm{Q}}} .
$$

By introducing a Dirac delta function, this integral can be brought to the equivalent form

$$
\mathrm{Z}_{\mathcal{D}}(\alpha, s)=\int_{0}^{s} \mathrm{~d} \phi_{1} \ldots \int_{0}^{s} \mathrm{~d} \phi_{\mathrm{Q}} \phi^{\alpha} \delta(\mathrm{s}-|\phi|)
$$


Moreover, owing to the Dirac delta function, all the upper integration limits can be pushed to infinity without changing the integral. If we replace the Dirac delta function by its Fourier representation

$$
\delta(z)=\frac{1}{2 \pi} \int_{-\infty}^{+\infty} \mathrm{d} \lambda \mathrm{e}^{-\mathrm{i} \lambda z},
$$

and rotate $\lambda \rightarrow \mathfrak{i} \lambda$, the integral turns into a complex one, performed along the imaginary axis, i.e.

$$
Z_{\mathcal{D}}(\alpha, s)=\frac{1}{2 \pi i} \int_{-i \infty}^{+i \infty} \mathrm{d} \lambda \mathrm{e}^{\lambda s}\left(\prod_{k=1}^{\mathrm{Q}} \int_{0}^{+\infty} \mathrm{d} \phi_{k} \phi_{k}^{\alpha_{k}-1} \mathrm{e}^{-\lambda \phi_{k}}\right),
$$

The inner integrals are Laplace transforms of monomials. They sum to

$$
\int_{0}^{+\infty} \mathrm{d} \phi \phi^{\alpha-1} \mathrm{e}^{-\lambda \phi}=\frac{\Gamma(\alpha)}{\lambda^{\alpha}} .
$$

Hence it follows

$$
\mathrm{Z}_{\mathcal{D}}(\alpha, s)=\frac{\prod_{\mathrm{k}=1}^{\mathrm{Q}} \Gamma\left(\alpha_{\mathrm{k}}\right)}{2 \pi \mathrm{i}} \int_{-\mathrm{i} \infty}^{+\mathrm{i} \infty} \mathrm{d} \lambda \frac{\mathrm{e}^{\lambda s}}{\lambda^{|\alpha|}}=\frac{\prod_{\mathrm{k}=1}^{\mathrm{Q}} \Gamma\left(\alpha_{\mathrm{k}}\right)}{\Gamma(|\alpha|)} \mathrm{s}^{|\alpha|-1},
$$

as a result of the Laplace antitransform of $\lambda^{-|\alpha|}$.

\section{A.2 Integrals of $V_{\alpha}$ and $U_{\alpha}$ on $\bar{T}_{Q}(s)$}

The integral of $V_{\alpha}$ on $\bar{T}_{Q}(s)$ follows trivially from a term-by-term integration of its monomial expansion, namely

$$
\mathrm{Z}_{\mathrm{V}}(\alpha, \mathrm{s}) \equiv \int_{\overline{\mathrm{T}}_{\mathrm{Q}}(\mathrm{s})} \mathrm{d} \bar{\phi} \mathrm{V}_{\alpha}(\bar{\phi})=\sum_{\beta \leqslant \alpha} v_{\alpha \beta}(\mathrm{k}) \frac{\prod_{\mathrm{m}=1}^{\mathrm{Q}-1} \Gamma\left(\beta_{\mathrm{m}}+1\right)}{\Gamma(|\beta|+\mathrm{Q})} .
$$

The integral of $\mathrm{U}_{\alpha}$ on $\overline{\mathrm{T}}_{\mathrm{Q}}(\mathrm{s})$ can be similarly calculated, provided we first represent it as a monomial sum. To this aim, we just need to apply the standard binomial formula

$$
\partial_{x}^{k}[f(x) g(x)]=\sum_{\ell=0}^{k}\left(\begin{array}{l}
k \\
l
\end{array}\right)\left[\partial_{x}^{k-\ell} f(x)\right]\left[\partial_{x}^{\ell} g(x)\right],
$$

in sequence to the various factors of eq. (2.7). We also observe that, given $k, m \in \mathbb{N}$ with $k \leqslant m$, it holds

$$
\begin{aligned}
& \partial_{x}^{k} x^{m}=k !\left(\begin{array}{c}
m \\
k
\end{array}\right) x^{m-k} \\
& \partial_{x}^{k}(s-x)^{m}=(-1)^{k} k !\left(\begin{array}{c}
m \\
k
\end{array}\right)(s-x)^{m-k} .
\end{aligned}
$$

Accordingly, we have

$$
\mathrm{U}_{\alpha}(\bar{\phi})=\frac{1}{\mathcal{D}_{\mathrm{K}}(\bar{\phi})} \partial_{2}^{\alpha_{2}} \ldots \partial_{\mathrm{Q}-1}^{\alpha_{\mathrm{Q}-1}} \mathrm{P}_{1}(\bar{\phi}) .
$$

with

$$
\begin{aligned}
\mathrm{P}_{1}(\bar{\phi}) & =\phi_{2}^{\alpha_{2}+\kappa_{2}-1} \ldots \phi_{\mathrm{Q}-1}^{\alpha_{\mathrm{Q}-1}+\kappa_{\mathrm{Q}-1}-1} \partial_{1}^{\alpha_{1}}\left\{\phi_{1}^{\alpha_{1}+\kappa_{1}-1}(s-|\bar{\phi}|)^{|\alpha|+\kappa_{\mathrm{Q}}-1}\right\} \\
& =\alpha_{1} ! \sum_{\eta_{1}=0}^{\alpha_{1}}(-1)^{\eta_{1}}\left(\begin{array}{c}
\alpha_{1}+\kappa_{1}-1 \\
\alpha_{1}-\eta_{1}
\end{array}\right)\left(\begin{array}{c}
|\alpha|+\kappa_{\mathrm{Q}}-1 \\
\eta_{1}
\end{array}\right) \\
& \cdot \phi_{1}^{\eta_{1}+\kappa_{1}-1} \phi_{2}^{\alpha_{2}+\kappa_{2}-1} \ldots \phi_{\mathrm{Q}-1}^{\alpha_{\mathrm{Q}-1}+\kappa_{\mathrm{Q}-1}-1}(s-|\bar{\phi}|)^{|\alpha|-\eta_{1}+\kappa_{\mathrm{Q}}-1} .
\end{aligned}
$$


Upon iterating the above calculation over all derivatives, we arrive at

$$
\begin{gathered}
\mathrm{u}_{\alpha}(\bar{\phi})=\left[\prod_{m=1}^{\mathrm{Q}-1} \Gamma\left(\alpha_{\mathrm{m}}+1\right)\right] \sum_{\eta \leqslant \alpha}(-1)^{|\eta|} \prod_{m=1}^{\mathrm{Q}-1}\left(\begin{array}{c}
\alpha_{\mathrm{m}}+\kappa_{\mathrm{m}}-1 \\
\alpha_{\mathrm{m}}-\beta_{\mathrm{m}}
\end{array}\right)\left(\begin{array}{c}
|\alpha|-\mathrm{L}_{\mathrm{m}}+\kappa_{\mathrm{Q}}-1 \\
\eta_{\mathrm{m}}
\end{array}\right) \\
\cdot \phi_{1}^{\eta_{1}} \ldots \phi_{\mathrm{Q}-1}^{\eta_{\mathrm{Q}-1}}(\mathrm{~s}-|\phi|)^{|\alpha|-|\eta|},
\end{gathered}
$$

with $\mathrm{L}_{1} \equiv 0$ and $\mathrm{L}_{\mathrm{m}} \equiv \sum_{\mathrm{k}=1}^{\mathrm{m}-1} \eta_{\mathrm{k}}$ for $\mathrm{m} \geqslant 2$. Therefore, we have

$$
\begin{aligned}
\mathrm{Z}_{\mathrm{u}}(\alpha, s) & \equiv \int_{\overline{\mathrm{T}}_{\mathrm{Q}}(\mathrm{s})} \mathrm{d} \bar{\phi} \mathrm{U}_{\alpha}(\bar{\phi})=\frac{\prod_{\mathrm{m}=1}^{\mathrm{Q}-1} \Gamma\left(\alpha_{\mathrm{m}}+1\right)}{\Gamma(|\alpha|+\mathrm{Q})} \\
& \cdot \sum_{\eta \leqslant \alpha}(-1)^{|\eta|} \Gamma(|\alpha|-|\eta|+1) \prod_{\mathrm{m}=1}^{\mathrm{Q}-1} \Gamma\left(\eta_{\mathrm{m}}+1\right)\left(\begin{array}{c}
\alpha_{\mathrm{m}}+\kappa_{\mathrm{m}}-1 \\
\alpha_{\mathrm{m}}-\eta_{\mathrm{m}}
\end{array}\right)\left(\begin{array}{c}
|\alpha|-\mathrm{L}_{\mathrm{m}}+\kappa_{\mathrm{Q}}-1 \\
\eta_{\mathrm{m}}
\end{array}\right) .
\end{aligned}
$$

\section{References}

[1] C. Castellano, S. Fortunato, and V. Loreto. Statistical physics of social dynamics. Rev. Mod. Phys., 81:591-646, May 2009.

[2] M. J. Keeling and K. T. D. Eames. Networks and epidemic models. J. R. Soc. Interface, 2:295, 2005.

[3] C. W. Gardiner. Handbook of Stochastic Methods. Springer Series in Synergetics. Springer, 1994.

[4] A. V. Skorokhod. Stochastic Equations for Diffusion Processes in a Bounded Region. II. Theory of Probability and its Applications, 7(1):3-23, 1962.

[5] A. Ern and J. Guermond. Theory and practice of finite elements, volume 159 of Applied Mathematical Sciences. Springer-Verlag, New York, 2004.

[6] J. Shen, T. Tang, and L. Wang. Spectral Methods: Algorithms, Analysis and Applications. Springer, 2011 edition, 2011.

[7] M. Mobilia, A. Petersen, and S. Redner. On the role of zealotry in the voter model. Journal of Statistical Mechanics: Theory and Experiment, 2007(08):P08029+, 2007.

[8] C. F. Dunkl and Y. Xu. Orthogonal Polynomials of Several Variables. Encyclopedia of Mathematics and its Applications. Cambridge University Press, 2001.

[9] C. de Boor. Computational aspects of multivariate polynomial interpolation: Indexing the coefficients. Advances in Computational Mathematics, 12(4):289-301, 2000.

[10] P. D. Lax and A. Milgram. Parabolic equations, contributions to the theory of partial differential equations. Annals of Mathematics Studies, (33):167-190, 1954.

[11] Y. Saad. Iterative Methods for Sparse Linear Systems. Society for Industrial and Applied Mathematics, 2 edition, April 2003.

[12] P. Clifford and A. Sudbury. A model for spatial conflict. Biometrika, 60(3):581-588, 1973.

[13] R. Holley and T. M. Liggett. Ergodic theorems for weakly interacting infinite systems and the voter model. The Annals of Probability, 3(4):643-663, 1975. 
[14] M. Mobilia. Does a single zealot affect an infinite group of voters? Physical Review Letters, 91:028701, 2003.

[15] M. Starnini, A. Baronchelli, and R. Pastor-Satorras. Ordering dynamics of the multi-state voter model. J. Stat. Mech., P10027, 2012, 1207.5810.

[16] S. Chen, H. Hu, J. Chen, and Z. Chen. Multi-state voter model on weighted social networks with committed agents. Int. J. Mod. Phys. C, 25:1450022, 2014.

[17] D. E. Knuth. The Art of Computer Programming, Volume 4A: Combinatorial Algorithms, volume 4A. Addison-Wesley Professional, 1 edition, January 2011.

[18] F. Palombi and S. Toti. Stochastic Dynamics of the Multi-State Voter Model over a Network based on Interacting Cliques and Zealot Candidates. Journal of Statistical Physics, 156:336-367, 2014.

[19] M. Girvan and M. E. J. Newman. Community structure in social and biological networks. Proceedings of the National Academy of Sciences, 99(12):7821-7826, 2002.

[20] J. Xie, J. Emenheiser, M. Kirby, S. Sreenivasan, B. K. Szymanski, and G. Korniss. Evolution of opinions on social networks in the presence of competing committed groups. PLoS ONE, 7(3):e33215, 2012.

[21] see http://www.cresco.enea.it/english for information. 